IJMMS 2003:5, 263-294

PII. S0161171203203185

http://ijmms.hindawi.com

(c) Hindawi Publishing Corp.

\title{
DYNAMICS OF A CLASS OF UNCERTAIN NONLINEAR SYSTEMS UNDER FLOW-INVARIANCE CONSTRAINTS
}

\author{
OCTAVIAN PASTRAVANU and MIHAIL VOICU
}

Received 18 March 2002

\begin{abstract}
For a class of uncertain nonlinear systems (UNSs), the flow-invariance of a timedependent rectangular set (TDRS) defines individual constraints for each component of the state-space trajectories. It is shown that the existence of the flowinvariance property is equivalent to the existence of positive solutions for some differential inequalities with constant coefficients (derived from the state-space equation of the UNS). Flow-invariance also provides basic tools for dealing with the componentwise asymptotic stability as a special type of asymptotic stability, where the evolution of the state variables approaching the equilibrium point (EP) $\{0\}$ is separately monitored (unlike the standard asymptotic stability, which relies on global information about the state variables, formulated in terms of norms). The $\mathrm{EP}\{0\}$ of a given UNS is proved to be componentwise asymptotically stable if and only if the $\mathrm{EP}\{0\}$ of a differential equation with constant coefficients is asymptotically stable in the standard sense. Supplementary requirements for the individual evolution of the state variables approaching the $\mathrm{EP}\{0\}$ allow introducing the stronger concept of componentwise exponential asymptotic stability, which can be characterized by algebraic conditions. Connections with the componentwise asymptotic stability of an uncertain linear system resulting from the linearization of a given UNS are also discussed.
\end{abstract}

2000 Mathematics Subject Classification: 34C11, 34D05, 93D20, 93C10, 93C41.

1. Introduction. Flow-invariance theory emerged from the pioneering research developed by Nagumo [8] and Hukuhara [4] at the middle of the preceding century, and further significant contributions have been brought by many well-known mathematicians, among which are Brezis [1], Crandall [2], and Martin [5]. Two remarkable monographs on this field are due to Pavel [11] and Motreanu and Pavel [7]. Voicu, in [12, 13], proposes the use of flowinvariant hyperrectangles for continuous-time linear systems, resulting in the definition and analysis of a special type of (exponential) asymptotic stability, namely, the componentwise (exponential) asymptotic stability. Later on, an overview on the applications of the flow-invariance method in control theory and design was presented in [14]. Recent results have extended these new concepts for linear systems with time-delay [3] and for linear systems with interval matrix [9]. Robustness problems for componentwise asymptotic stability have been addressed in [10]. 
The current paper focuses on a class of nonlinear systems with uncertainties and uses the powerful tool offered by the flow-invariance theory to reveal some important properties of state trajectories around the equilibrium points, which remain unexplored within the standard framework of stability analysis. These properties allow a componentwise refinement of the dynamics, and, consequently, they present a particular interest for real-life engineering problems, where individual information about the evolution of each state variable is more valuable than a global characterization of trajectories (expressed in terms of a certain norm). Moreover, our results are able to cover a whole family of nonlinear systems, corresponding to the uncertainties that can affect the model construction.

The exposition gradually displaces its gravity center from the qualitative analysis of the time-dependent rectangular sets which are flow invariant with respect to the nonlinear uncertain system towards the componentwise (exponential) asymptotic stability of the equilibrium point according to the following plan. Section 2 deals with the existence of flow-invariant time-dependent rectangular sets constraining the state trajectories and prepares the background for a detailed exploration of the whole family of such hyperrectangles that are flow-invariant with respect to a given nonlinear uncertain system (Section 3). Componentwise asymptotic stability and componentwise exponential asymptotic stability are addressed in Sections 4 and 5, respectively. In Section 6, the componentwise asymptotic stability for linear approximation is discussed, and Section 7 illustrates the overall approach by two examples commented adequately.

Taking into consideration the mathematical nature of the problems raised by the flow-invariance method, we are going to use componentwise (elementby-element) matrix inequalities $\mathbf{P}<\mathbf{Q}$ and $\mathbf{P} \leq \mathbf{Q}, \mathbf{P}, \mathbf{Q} \in \mathbb{R}^{n \times m}$ meaning for all $i=1, \ldots, n$, for all $j=1, \ldots, m,(\mathbf{P})_{i j}<(\mathbf{Q})_{i j}$, and $(\mathbf{P})_{i j} \leq(\mathbf{Q})_{i j}$, respectively. These notations preserve their signification when handling vectors or vector functions.

2. Flow-invariance property of the free response. Consider the class of uncertain nonlinear systems (UNSs) defined as

$$
\begin{gathered}
\dot{\mathbf{x}}=\mathbf{f}(\mathbf{x}), \quad \mathbf{x} \in \mathbb{R}^{n}, \quad \mathbf{x}\left(t_{0}\right)=\mathbf{x}_{0}, \quad t \geq t_{0} ; \\
f_{i}(\mathbf{x})=\sum_{j=1}^{n} a_{i j} x_{j}^{p_{i j}}, \quad p_{i j} \in \mathbb{N}, i=1, \ldots, n,
\end{gathered}
$$

where the interval-type coefficients

$$
a_{i j}^{-} \leq a_{i j} \leq a_{i j}^{+}
$$

are chosen to cover the inherent errors which frequently affect the accuracy of model construction. For any concrete value of the coefficients $a_{i j}$, belonging 
to the intervals (2.2), the Cauchy problem associated to UNS (2.1) has a unique local solution for any $t_{0}$ and $\mathbf{x}\left(t_{0}\right)=\mathbf{x}_{0}$ since the vector function $\mathbf{f}(\mathbf{x})$ fulfills the local Lipschitz condition.

We also consider the $n$-valued vector function $\boldsymbol{\gamma}(t)$, with differentiable and positive components $\gamma_{i}(t)>0, i=1, \ldots, n$. Using these $\gamma_{i}(t)>0, i=1, \ldots, n$, define a time-dependent rectangular set (TDRS)

$$
\mathbf{H}_{\boldsymbol{\gamma}}(t)=\left[-\gamma_{1}(t), \gamma_{1}(t)\right] \times \cdots \times\left[-\gamma_{n}(t), \gamma_{n}(t)\right],
$$

where $[] \times,[$,$] denotes the Cartesian product.$

We are now interested in exploring the free response of UNS (2.1) along the lines of the componentwise constrained evolution of the state trajectories induced by the concept of flow-invariance (FI) [7, 11].

DEFINITION 2.1. TDRS (2.3) is FI with respect to UNS (2.1) if there exists $T>t_{0}$ such that for any initial condition $\mathbf{x}\left(t_{0}\right)=\mathbf{x}_{0} \in \mathbf{H}_{\boldsymbol{\gamma}}\left(t_{0}\right)$, the corresponding state trajectory $\mathbf{x}(t)=\mathbf{x}\left(t ; t_{0}, x_{0}\right)$ remains (for all possible values resulting from the interval-type coefficients) inside $\mathbf{H}_{\boldsymbol{\gamma}}(t)$, for $t \in\left[t_{0}, T\right)$, that is,

$$
\exists T>t_{0}, \quad \forall \mathbf{x}\left(t_{0}\right)=\mathbf{x}_{0} \in \mathbf{H}_{\boldsymbol{\gamma}}\left(t_{0}\right), \quad \mathbf{x}(t)=\mathbf{x}\left(t ; t_{0}, \mathbf{x}_{0}\right) \in \mathbf{H}_{\boldsymbol{\gamma}}(t), \quad t \in\left[t_{0}, T\right) .
$$

THEOREM 2.2. TDRS (2.3) is FI with respect to UNS (2.1) if and only if the following inequalities hold for $t \in\left[t_{0}, T\right), T>t_{0}$ :

$$
\begin{array}{lll}
\dot{\boldsymbol{\gamma}}(t) \geq \overline{\mathbf{g}}(\boldsymbol{\gamma}) ; \quad \overline{\mathbf{g}}: \mathbb{R}^{n} \longrightarrow \mathbb{R}^{n}, & \overline{\mathfrak{g}}_{i}(\boldsymbol{\gamma})=\sum_{j=1}^{n} \bar{c}_{i j} \gamma_{j}^{p_{i j}}, \quad i=1, \ldots, n, \\
\dot{\boldsymbol{\gamma}}(t) \geq \tilde{\mathbf{g}}(\boldsymbol{\gamma}) ; \quad \tilde{\mathbf{g}}: \mathbb{R}^{n} \longrightarrow \mathbb{R}^{n}, & \tilde{g}_{i}(\boldsymbol{\gamma})=\sum_{j=1}^{n} \tilde{c}_{i j} \gamma_{j}^{p_{i j}}, \quad i=1, \ldots, n,
\end{array}
$$

where $\bar{c}_{i j}$ and $\widetilde{c}_{i j}$ have unique values derived from the interval-type coefficients $a_{i j}$ of UNS (2.1) as follows:

$$
\begin{aligned}
& \bar{c}_{i j}=a_{i i}^{+}, \quad \text { for } p_{i i} \text { odd or even; } \quad \bar{c}_{i j}= \begin{cases}\max \left\{\left|a_{i j}^{-}\right|,\left|a_{i j}^{+}\right|\right\}, & \text {if } p_{i j} \text { odd, } \\
\max \left\{0, a_{i j}^{+}\right\}, & \text {if } p_{i j} \text { even; }\end{cases} \\
& \tilde{c}_{i j}=\left\{\begin{array}{ll}
a_{i i}^{+}, & \text {if } p_{i i} \text { odd, } \\
-a_{i i}^{-}, & \text {if } p_{i i} \text { even; }
\end{array} \quad \tilde{c}_{i j}= \begin{cases}\max \left\{\left|a_{i j}^{-}\right|,\left|a_{i j}^{+}\right|\right\}, & \text {if } p_{i j} \text { odd, } \\
\max \left\{0,-a_{i j}^{-}\right\}, & \text {if } p_{i j} \text { even. }\end{cases} \right.
\end{aligned}
$$

Proof. A necessary and sufficient condition for $\operatorname{TDRS} \mathbf{H}_{\boldsymbol{\gamma}}(t)$ in (2.3) to be FI with respect to UNS (2.1) can be formulated, according to [11, pages 74-75], 
as follows:

$$
\begin{gathered}
\sum_{\substack{j=1 \\
j \neq i}}^{n} a_{i j} v_{j}^{p_{i j}}(t)+a_{i i} \gamma_{i}^{p_{i i}}(t) \leq \dot{\gamma}_{i}(t), \quad i=1, \ldots, n, \\
-\dot{\gamma}_{i}(t) \leq \sum_{\substack{j=1 \\
j \neq i}}^{n} a_{i j} v_{j}^{p_{i j}}(t)+a_{i i}(-1)^{p_{i i}} \gamma_{i}^{p_{i i}}(t), \quad i=1, \ldots, n,
\end{gathered}
$$

for

$$
-\gamma_{i}(t) \leq v_{i}(t) \leq \gamma_{i}(t), \quad t \in\left[t_{0}, T\right), i=1, \ldots, n .
$$

For $i \neq j$ and $p_{i j}$ odd, we can write

$$
-\max \left\{\left|a_{i j}^{-}\right|,\left|a_{i j}^{+}\right|\right\} \gamma_{j}^{p_{i j}}(t) \leq a_{i j} v_{j}^{p_{i j}}(t) \leq \max \left\{\left|a_{i j}^{-}\right|,\left|a_{i j}^{+}\right|\right\} \gamma_{j}^{p_{i j}}(t),
$$

and, similarly, for $i \neq j$ and $p_{i j}$ even,

$$
-\max \left\{-a_{i j}^{-}, 0\right\} \gamma_{j}^{p_{i j}}(t) \leq a_{i j} v_{j}^{p_{i j}}(t) \leq \max \left\{a_{i j}^{+}, 0\right\} \gamma_{j}^{p_{i j}}(t) .
$$

For $i=j$ and $p_{i i}$ odd, we have

$$
a_{i i} \gamma_{i}^{p_{i i}}(t) \leq a_{i i}^{+} \gamma_{i}^{p_{i i}}(t), \quad-a_{i i}^{+} \gamma_{i}^{p_{i i}}(t) \leq-a_{i i} \gamma_{i i}^{p_{i i}}(t)=a_{i i}\left(-\gamma_{i}(t)\right)^{p_{i i}}
$$

For $i=j$ and $p_{i i}$ even, we have

$$
\begin{gathered}
a_{i i} \gamma_{i}^{p_{i i}}(t) \leq a_{i i}^{+} \gamma_{i}^{p_{i i}}(t), \\
-\left(-a_{i i}^{-}\right) \gamma_{i}^{p_{i i}}(t)=a_{i i}^{-} \gamma_{i}^{p_{i i}}(t) \leq a_{i i} \gamma_{i i}^{p_{i i}}(t)=a_{i i}\left(-\gamma_{i}(t)\right)^{p_{i i}} .
\end{gathered}
$$

The fulfillment of the first set of differential inequalities formulated above means

$$
\begin{aligned}
& \sum_{\substack{j=1 \\
j \neq i \\
p_{i j} \text { odd }}}^{n} \max \left\{\left|a_{i j}^{-}\right|,\left|a_{i j}^{+}\right|\right\} \gamma_{j}^{p_{i j}}(t) \\
& \quad+\sum_{\substack{j=1 \\
j \neq i \\
p_{i j} \text { even }}}^{n} \max \left\{a_{i j}^{+}, 0\right\} \gamma_{j}^{p_{i j}}(t)+a_{i i}^{+} \gamma_{i}^{p_{i i}}(t) \leq \dot{\gamma}_{i}(t), \quad i=1, \ldots, n,
\end{aligned}
$$

which is identical to (2.5) with coefficients (2.7). 
The fulfillment of the second set of differential inequalities formulated above means

$$
\begin{aligned}
& \sum_{\substack{j=1 \\
j \neq i \\
p_{i j} \text { odd }}}^{n} \max \left\{\left|a_{i j}^{-}\right|,\left|a_{i j}^{+}\right|\right\} \gamma_{j}^{p_{i j}}(t) \\
& \quad+\sum_{\substack{j=1 \\
j \neq i \\
p_{i j} \text { even }}}^{n} \max \left\{-a_{i j}^{-}, 0\right\} \gamma_{j}^{p_{i j}}(t)+\phi_{i i} \gamma_{i}^{p_{i i}}(t) \leq \dot{\gamma}_{i}(t), \quad i=1, \ldots, n,
\end{aligned}
$$

where

$$
\phi_{i i}= \begin{cases}a_{i i}^{+}, & \text {if } p_{i i} \text { odd } \\ -a_{i i}^{-}, & \text {if } p_{i i} \text { even }\end{cases}
$$

which is identical to (2.6) with coefficients (2.8).

THEOREM 2.3. There exist TDRSs (2.3) which are FI with respect to UNS (2.1) if and only if there exist common positive solutions (PSS) for the following differential inequalities (DIs):

$$
\dot{\mathbf{y}} \geq \overline{\mathbf{g}}(\mathbf{y}), \quad \dot{\mathbf{y}} \geq \tilde{\mathbf{g}}(\mathbf{y}) .
$$

Proof. It is a direct consequence of Definition 2.1 and Theorem 2.2.

THEOREM 2.4. There exist TDRSs (2.3) which are FI with respect to UNS (2.1) if and only if there exist PSs for the following DI:

$$
\dot{\mathbf{y}} \geq \mathbf{g}(\mathbf{y}) ; \quad \mathbf{g}: \mathbb{R}^{n} \longrightarrow \mathbb{R}^{n}, \quad g_{i}(\mathbf{y})=\max _{\mathbf{y} \in \mathbf{R}^{n}}\left\{\bar{g}_{i}(\mathbf{y}), \tilde{g}_{i}(\mathbf{y})\right\}, \quad i=1, \ldots, n .
$$

PRoof. DI (2.19) replaces the two DIs (2.18) from Theorem 2.3 in an equivalent manner.

3. The family of flow-invariant TDRSs. In order to investigate the family of TDRSs, which are FI with respect to a given UNS, we will first focus on some relevant characteristics of the PSs of DI (2.19) since Theorem 2.4 emphasizes a bijective link between the two types of mathematical objects. We start with the qualitative exploration of the solution of the following differential equation (DE):

$$
\dot{\mathbf{z}}=\mathbf{g}(\mathbf{z}) \text {, }
$$

which is obtained from DI (2.19) by replacing " $\geq$ " with "=".

LEMMA 3.1. $D E$ (3.1), with arbitrary $t_{0}$ and arbitrary initial condition $\mathbf{z}\left(t_{0}\right)=$ $\mathbf{z}_{0}$, has a unique solution $\mathbf{z}(t)=\mathbf{z}\left(t ; t_{0}, \mathbf{z}_{0}\right)$ defined on $\left[t_{0}, T\right)$ for some $T>t_{0}$. 
Proof. We prove that $\mathbf{g}(z)$, defined according to (2.19), fulfills the Lipschitz condition.

Both $\bar{g}_{i}$ and $\tilde{g}_{i}, i=1, \ldots, n$, can be written by separating the even and odd powers as follows:

$$
\overline{\mathfrak{g}}_{i}(\mathbf{z})=\bar{\varphi}_{i}(\mathbf{z})+\bar{\psi}_{i}(\mathbf{z}), \quad \widetilde{\mathfrak{g}}_{i}(\mathbf{z})=\widetilde{\varphi}_{i}(\mathbf{z})+\widetilde{\psi}_{i}(\mathbf{z})
$$

where

$$
\begin{array}{ll}
\bar{\varphi}_{i}(\mathbf{z})=\sum_{\substack{j=1 \\
p_{i j} \text { odd }}}^{n} \bar{c}_{i j} z_{j}^{p_{i j} ;} & \widetilde{\varphi}_{i}(\mathbf{z})=\sum_{\substack{j=1 \\
p_{i j} \text { odd }}}^{n} \widetilde{c}_{i j} z_{j}^{p_{i j}}, \\
\bar{\psi}_{i}(\mathbf{z})=\sum_{\substack{j=1 \\
p_{i j} \text { even }}}^{n} \bar{c}_{i j} z_{j}^{p_{i j} ;} & \widetilde{\psi}_{i}(\mathbf{z})=\sum_{\substack{j=1 \\
p_{i j} \text { even }}}^{n} \widetilde{c}_{i j} z_{j}^{p_{i j}} .
\end{array}
$$

Based on the expression derived in Theorem 2.2 for coefficients $\bar{c}_{i j}(2.7)$ and $\tilde{c}_{i j}(2.8)$, it results that $\bar{\varphi}_{i}(\mathbf{z})$ and $\tilde{\varphi}_{i}(\mathbf{z})$ are identical, and, therefore, both of them can be replaced by a unique function

$$
\bar{\varphi}_{i}(\mathbf{z})=\tilde{\varphi}_{i}(\mathbf{z})=: \varphi_{i}(\mathbf{z}), \quad i=1, \ldots, n .
$$

Thus, we get

$$
g_{i}(\mathbf{z})=\max _{\mathbf{z} \in \mathbb{R}^{n}}\left\{\varphi_{i}(\mathbf{z})+\bar{\psi}_{i}(\mathbf{z}), \varphi_{i}(\mathbf{z})+\widetilde{\psi}_{i}(\mathbf{z})\right\}=\varphi_{i}(\mathbf{z})+\max _{\mathbf{z} \in \mathbb{R}^{n}}\left\{\bar{\psi}_{i}(\mathbf{z}), \widetilde{\psi}_{i}(\mathbf{z})\right\}
$$

Denote by $\psi_{i}(z)$ the function defined as

$$
\psi_{i}(\mathbf{z})=\max _{\mathbf{z} \in \mathbb{R}^{n}}\left\{\bar{\psi}_{i}(\mathbf{z}), \widetilde{\psi}_{i}(\mathbf{z})\right\}
$$

Hence, we can write

$$
g_{i}(\mathbf{z})=\varphi_{i}(\mathbf{z})+\psi_{i}(\mathbf{z}), \quad i=1, \ldots, n,
$$

and, consequently, the vector function $\mathbf{g}$ is given by

$$
\mathbf{g}(\mathbf{z})=\boldsymbol{\varphi}(\mathbf{z})+\boldsymbol{\psi}(\mathbf{z})
$$

Function $\boldsymbol{\varphi ( z )}$ satisfies the Lipschitz condition. In order to have the Lipschitz property for $\mathbf{g}(\mathbf{z})$, we have to show that $\boldsymbol{\psi}(\mathbf{z})$ also fulfills the Lipschitz condition.

For arbitrary $\mathbf{x}, \mathbf{y} \in \mathbf{K}\left(\mathbf{K} \subset \mathbb{R}^{n}\right.$ a compact set), we have

$$
\|\boldsymbol{\Psi}(\mathbf{x})-\boldsymbol{\psi}(\mathbf{y})\|_{2}^{2}=\sum_{i=1}^{n}\left|\psi_{i}(\mathbf{x})-\psi_{i}(\mathbf{y})\right|^{2},
$$


where

$$
\left|\psi_{i}(\mathbf{x})-\psi_{i}(\mathbf{y})\right|=\left|\max _{\mathbf{x} \in \mathbf{K}}\left\{\bar{\psi}_{i}(\mathbf{x}), \widetilde{\psi}_{i}(\mathbf{x})\right\}-\max _{\mathbf{y} \in \mathbf{K}}\left\{\bar{\psi}_{i}(\mathbf{y}), \widetilde{\psi}_{i}(\mathbf{y})\right\}\right| .
$$

Both $\bar{\psi}_{i}(\mathbf{z})$ and $\widetilde{\psi}_{i}(\mathbf{z})$ meet the Lipschitz condition on $\mathbf{K}$. Hence, there exist $\bar{L}_{i}>0$ and $\widetilde{L}_{i}>0$ such that

$$
\begin{array}{ll}
\forall \mathbf{x}, \mathbf{y} \in \mathbf{K}, & \left|\bar{\psi}_{i}(\mathbf{x})-\bar{\psi}_{i}(\mathbf{y})\right| \leq \bar{L}_{i}\|\mathbf{x}-\mathbf{y}\|_{2}, \\
\forall \mathbf{x}, \mathbf{y} \in \mathbf{K}, & \left|\tilde{\psi}_{i}(\mathbf{x})-\tilde{\psi}_{i}(\mathbf{y})\right| \leq \widetilde{L}_{i}\|\mathbf{x}-\mathbf{y}\|_{2} .
\end{array}
$$

Thus, there exists a positive constant $L_{i}=\max \left\{\bar{L}_{i}, \widetilde{L}_{i}\right\}$ such that

$$
\forall \mathbf{x}, \mathbf{y} \in \mathbf{K}, \quad\left|\bar{\psi}_{i}(\mathbf{x})-\bar{\psi}_{i}(\mathbf{y})\right| \leq L_{i}\|\mathbf{x}-\mathbf{y}\|_{2}, \quad\left|\tilde{\psi}_{i}(\mathbf{x})-\tilde{\psi}_{i}(\mathbf{y})\right| \leq L_{i}\|\mathbf{x}-\mathbf{y}\|_{2}
$$

On the other hand, the compact set $\mathbf{K}$ can be regarded as a union of the subsets

$$
\mathbf{K}=\overline{\mathbf{K}} \cup \widetilde{\mathbf{K}} \cup \hat{\mathbf{K}}
$$

where

$$
\begin{aligned}
& \overline{\mathbf{K}}=\left\{\mathbf{z} \in \mathbf{K} \mid \bar{\psi}_{i}(\mathbf{z})>\widetilde{\psi}_{i}(\mathbf{z})\right\}, \\
& \widetilde{\mathbf{K}}=\left\{\mathbf{z} \in \mathbf{K} \mid \bar{\psi}_{i}(\mathbf{z})<\widetilde{\psi}_{i}(\mathbf{z})\right\}, \\
& \hat{\mathbf{K}}=\left\{\mathbf{z} \in \mathbf{K} \mid \bar{\psi}_{i}(\mathbf{z})=\widetilde{\psi}_{i}(\mathbf{z})\right\} .
\end{aligned}
$$

Obviously, we are interested in exploring the general case when $\overline{\mathbf{K}}, \tilde{\mathbf{K}}$, and $\hat{\mathbf{K}}$ are nonempty, which covers all other possible situations.

When $\mathbf{x}, \mathbf{y}$ belong to the same subset, and $\psi_{i}(\mathbf{x})$ and $\psi_{i}(\mathbf{y})$ are defined by the same function (i.e., either $\bar{\psi}_{i}$ or $\tilde{\psi}_{i}$ ), we, therefore, can write

$$
\left|\psi_{i}(\mathbf{x})-\psi_{i}(\mathbf{y})\right|<L_{i}\|\mathbf{x}-\mathbf{y}\|_{2} .
$$

We deal with the other cases when $\mathbf{x}$ and $\mathbf{y}$ belong to different subsets.

(1) For $\mathbf{x} \in \overline{\mathbf{K}}, \mathbf{y} \in \tilde{\mathbf{K}}$, we have

$$
\left|\psi_{i}(\mathbf{x})-\psi_{i}(\mathbf{y})\right|=\left|\bar{\psi}_{i}(\mathbf{x})-\tilde{\psi}_{i}(\mathbf{y})\right|
$$

which means one of the following two situations:

(1a) $\bar{\psi}_{i}(\mathbf{x}) \geq \widetilde{\psi}_{i}(\mathbf{y})$ implies $\left|\psi_{i}(\mathbf{x})-\psi_{i}(\mathbf{y})\right|=\bar{\psi}_{i}(\mathbf{x})-\widetilde{\psi}_{i}(\mathbf{y})$.

On the other hand, $\mathbf{y} \in \tilde{\mathbf{K}} \Rightarrow \tilde{\psi}_{i}(\mathbf{y})>\bar{\psi}_{i}(\mathbf{y})$.

Thus, we conclude that $\left|\psi_{i}(\mathbf{x})-\psi_{i}(\mathbf{y})\right|<\bar{\psi}_{i}(\mathbf{x})-\bar{\psi}_{i}(\mathbf{y}) \leq L_{i}\|\mathbf{x}-\mathbf{y}\|_{2}$.

(1b) $\bar{\psi}_{i}(\mathbf{x})<\tilde{\psi}_{i}(\mathbf{y})$ implies $\left|\psi_{i}(\mathbf{x})-\psi_{i}(\mathbf{y})\right|=\tilde{\psi}_{i}(\mathbf{y})-\bar{\psi}_{i}(\mathbf{x})$.

On the other hand, $\mathbf{x} \in \overline{\mathbf{K}} \Rightarrow \bar{\psi}_{i}(\mathbf{x})>\widetilde{\psi}_{i}(\mathbf{x})$.

Thus, we conclude that $\left|\psi_{i}(\mathbf{x})-\psi_{i}(\mathbf{y})\right|<\tilde{\psi}_{i}(\mathbf{y})-\widetilde{\psi}_{i}(\mathbf{x}) \leq L_{i}\|\mathbf{x}-\mathbf{y}\|_{2}$. 
(2) For $\mathbf{x} \in \overline{\mathbf{K}}, \mathbf{y} \in \hat{\mathbf{K}}$, we have

$$
\left|\psi_{i}(\mathbf{x})-\psi_{i}(\mathbf{y})\right|=\left|\bar{\psi}_{i}(\mathbf{x})-\bar{\psi}_{i}(\mathbf{y})\right| \leq L_{i}\|\mathbf{x}-\mathbf{y}\|_{2} .
$$

For the remaining cases $(\mathbf{x} \in \hat{\mathbf{K}}, \mathbf{y} \in \widetilde{\mathbf{K}} ; \mathbf{x} \in \widetilde{\mathbf{K}}, \mathbf{y} \in \overline{\mathbf{K}} ; \mathbf{x} \in \hat{\mathbf{K}}, \mathbf{y} \in \overline{\mathbf{K}})$, the approach is similar, and, consequently, we get

$$
\forall \mathbf{x}, \mathbf{y} \in \mathbf{K}, \quad\left|\psi_{i}(\mathbf{x})-\psi_{i}(\mathbf{y})\right| \leq L_{i}\|\mathbf{x}-\mathbf{y}\|_{2}, \quad i=1, \ldots, n
$$

Thus, for the vector function $\boldsymbol{\psi}$, we can write

$$
\begin{aligned}
\|\boldsymbol{\psi}(\mathbf{x})-\boldsymbol{\psi}(\mathbf{y})\|_{2}^{2} & =\sum_{i=1}^{n}\left|\psi_{i}(\mathbf{x})-\psi_{i}(\mathbf{y})\right|^{2} \leq \sum_{i=1}^{n}\left(L_{i}^{2}\|\mathbf{x}-\mathbf{y}\|_{2}^{2}\right) \\
& =\left(\sum_{i=1}^{n} L_{i}^{2}\right)\|\mathbf{x}-\mathbf{y}\|_{2}^{2},
\end{aligned}
$$

which means

$$
\forall \mathbf{x}, \mathbf{y} \in \mathbf{K}, \quad\|\boldsymbol{\psi}(\mathbf{x})-\boldsymbol{\psi}(\mathbf{y})\|_{2} \leq \sqrt{\sum_{i=1}^{n} L_{i}^{2}}\|\mathbf{x}-\mathbf{y}\|_{2},
$$

showing that $\boldsymbol{\psi}(\mathbf{z})$ satisfies the Lipschitz condition. Consequently, $\mathbf{g}(\mathbf{z})$ satisfies the Lipschitz condition.

This completes the proof for the existence and uniqueness of the solution of the Cauchy problem.

LEMMA 3.2. For any $t_{0}$ and any positive initial condition $\mathbf{z}\left(t_{0}\right)=\mathbf{z}_{0}>0$, the unique solution $\mathbf{z}(t)=\mathbf{z}\left(t ; t_{0}, \mathbf{z}_{0}\right)$ of $D E$ (3.1) remains positive for its maximal interval of existence $\left[t_{0}, T\right)$.

Proof. First, we prove that, for any $t_{0}$ and any nonnegative initial condition $\mathbf{z}\left(t_{0}\right)=\mathbf{z}_{0} \geq 0$, the unique solution $\mathbf{z}(t)=\mathbf{z}\left(t ; t_{0}, \mathbf{z}_{0}\right)$ of DE (3.1) remains nonnegative as long as it exists.

The uniqueness of $\mathbf{z}(t)$ is guaranteed by Lemma 3.1.

On the other hand, for any $\mathbf{z} \geq 0$, the definition of $g_{i}$ in (2.19) ensures the fulfillment of the inequality

$$
0 \leq g_{i}(\mathbf{z}) \text { with } z_{i}=0, z_{j} \geq 0, i \neq j \text {, for } i=1, \ldots, n \text {. }
$$

This means that, for the vector function $\mathbf{v}(t)=\left[v_{1}(t) \cdots v_{n}(t)\right]^{\prime}=[0 \cdots 0]^{\prime}$, where $[\cdots]^{\prime}$ denotes transposition, the inequality

$$
\dot{v}_{i}(t) \leq g_{i}\left(z_{1}, \ldots, z_{i-1}, v_{i}(t), z_{i+1}, \ldots, z_{n}\right), \quad i=1, \ldots, n
$$


holds for arbitrary nonnegative $\mathbf{z} \geq 0$, which is a necessary and sufficient condition for the flow invariance of the set $\mathbb{R}_{+}^{n}$ with respect to DE (3.1), that is,

$$
\forall t_{0} \leq t, \quad \forall \mathbf{z}\left(t_{0}\right)=\mathbf{z}_{0} \geq 0, \mathbf{z}\left(t ; t_{0}, \mathbf{z}_{0}\right) \geq 0
$$

Now, for any $t_{0}$ and any positive initial condition $\mathbf{z}\left(t_{0}\right)=\mathbf{z}_{0}>0$, we can write the following inequalities for the corresponding unique solution $\mathbf{z}(t)=$ $\mathbf{z}\left(t ; t_{0}, \mathbf{z}_{0}\right)$ of $\mathrm{DE}(3.1)$ :

$$
\dot{z}_{i}(t) \geq \hat{c}_{i i} z_{i}^{p_{i i}}(t), \quad \hat{c}_{i i}=\max \left\{\bar{c}_{i i}, \widetilde{c}_{i i}\right\}, i=1, \ldots, n,
$$

because, according to the definition of $g_{i}$ in (2.19), all the coefficients $\bar{c}_{i j}, \widetilde{c}_{i j}$, $j \neq i, j=1, \ldots, n$, are nonnegative, and all $z_{j}(t)$ are also nonnegative (from the first part of the current proof).

We start with the case when $\hat{c}_{i i} \geq 0$. As $z_{i}(t) \geq 0$ (from the first part of the current proof), we have

$$
\dot{z}_{i}(t) \geq 0, \quad z_{i}\left(t_{0}\right)>0,
$$

which shows that $z_{i}(t)$ is nondecreasing as long as it exists, yielding

$$
z_{i}(t) \geq z_{i}\left(t_{0}\right)>0
$$

for its maximal interval of existence.

Now, we deal with the case when $\hat{c}_{i i}<0$. Consider the differential equation

$$
\dot{r}_{i}(t)=\hat{c}_{i i} r_{i}^{p_{i i}}(t)
$$

with the initial condition

$$
r_{i}\left(t_{0}\right)=z_{i}\left(t_{0}\right)>0
$$

According to a well-known property of the scalar differential inequalities (e.g., [6, page 57]), we have

$$
z_{i}(t) \geq r_{i}(t), \quad t \in\left[t_{0}, T\right)
$$

where $\left[t_{0}, T\right)$ denotes the maximal interval of existence for both $z_{i}(t)$ and $r_{i}(t)$.

For $p_{i i}=1, r_{i}(t)$ is given by

$$
r_{i}(t)=z_{i}\left(t_{0}\right) e^{\hat{c}_{i i}\left(t-t_{0}\right)}, \quad t \in\left[t_{0}, \infty\right)
$$

and, therefore,

$$
z_{i}(t) \geq z_{i}\left(t_{0}\right) e^{\hat{c}_{i i}\left(t-t_{0}\right)}>0
$$

for the maximal interval of existence of $z_{i}(t)$. 
For $p_{i i} \geq 2, r_{i}(t)$ is given by

$$
r_{i}(t)=\frac{1}{\sqrt[p_{i i}-1]{-\hat{c}_{i i}\left(p_{i i}-1\right)\left(t-t_{0}\right)+1 / z_{i}^{p_{i-1}}\left(t_{0}\right)}}, \quad t \in\left[t_{0}, \infty\right),
$$

and, therefore,

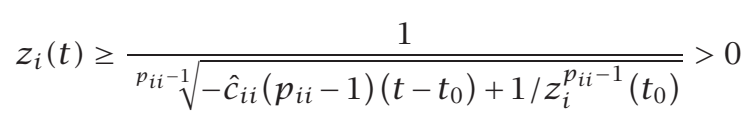

for the maximal interval of existence of $z_{i}(t)$. The proof is completed since $t_{0}$ and $\mathbf{z}\left(t_{0}\right)=\mathbf{z}_{0}>0$ were arbitrarily taken.

We can easily see that Lemma 3.2 guarantees the existence of PSs for DI (2.19) in the particular case when " $\geq$ " is replaced by "=." However, DI (2.19) might have PSs that do not satisfy DE (3.1), and, therefore, we further establish a connection between the PSs of DI (2.19) and the PSs of DE (3.1).

LEMMA 3.3. Let $\mathbf{y}(t)>0$ be an arbitrary PS of DI (2.19) with the maximal interval of existence $\left[t_{0}, T\right)$. Denote by $\mathbf{z}(t)$ an arbitrary PS of DE (3.1), corresponding to an initial condition $\mathbf{z}\left(t_{0}\right)$ that satisfies the componentwise inequality

$$
0<\mathbf{z}\left(t_{0}\right) \leq \mathbf{y}\left(t_{0}\right)
$$

Denote by $\mathbf{z}^{*}(t)$ the unique PS of DE (3.1) corresponding to the initial condition taken by $\mathbf{y}(t)$, that is,

$$
\mathbf{z}^{*}\left(t_{0}\right) \equiv \mathbf{y}\left(t_{0}\right)
$$

For $t \in\left[t_{0}, T\right)$, the following inequalities hold:

$$
0<\mathbf{z}(t) \leq \mathbf{z}^{*}(t) \leq \mathbf{y}(t)
$$

Proof. The fulfillment of the inequality $0<\mathbf{z}(t)$ for $0<\mathbf{z}\left(t_{0}\right)$ is guaranteed by Lemma 3.2. Suppose that there exists a vector function $\mathbf{h}(t) \in \mathbf{R}^{n}$, which is differentiable and positive for $t \in\left[t_{0}, T\right)$, with the following property: any solution of DE (3.1) $\mathbf{z}(t)=\mathbf{z}\left(t ; t_{0}, \mathbf{z}_{0}\right)$, whose initial condition satisfies the inequality

$$
0<\mathbf{z}\left(t_{0}\right)=\mathbf{z}_{0} \leq \mathbf{h}\left(t_{0}\right)
$$

satisfies the inequality

$$
0<\mathbf{z}(t)=\mathbf{z}\left(t ; t_{0}, \mathbf{z}_{0}\right) \leq \mathbf{h}(t), \quad t \in\left[t_{0}, T\right)
$$


According to [11, pages 74-75], a necessary and sufficient condition for such a property to take place is that

$$
g_{i}\left(z_{1}, \ldots, z_{i-1}, h_{i}, z_{i+1}, \ldots, z_{n}\right) \leq \dot{h}_{i}, \quad i=1, \ldots, n,
$$

for all $\mathbf{z}$ with $0<\mathbf{z} \leq \mathbf{h}$.

Using the definition of $g_{i}$ in (2.19), we can write, for $0<\mathbf{z} \leq \mathbf{h}(t)$,

$$
\begin{aligned}
& g_{i}\left(z_{1}, \ldots, z_{i-1}, h_{i}, z_{i+1}, \ldots, z_{n}\right) \\
& =\max _{h_{i}, z_{j}, j \neq i}\left\{\bar{c}_{i i} h_{i}^{p_{i i}}+\sum_{\substack{j=1 \\
j \neq 1}}^{n} \bar{c}_{i j} z_{j}^{p_{i j}}, \widetilde{c}_{i i} h_{i}^{p_{i i}}+\sum_{\substack{j=1 \\
j \neq 1}}^{n} \tilde{c}_{i j} z_{j}^{p_{i j}}\right\} \\
& \quad \leq \max _{\mathbf{h} \in \mathbb{R}^{n}}\left\{\bar{c}_{i i} h_{i}^{p_{i i}}+\sum_{\substack{j=1 \\
j \neq 1}}^{n} \bar{c}_{i j} h_{j}^{p_{i j}}, \widetilde{c}_{i i} h_{i}^{p_{i i}}+\sum_{\substack{j=1 \\
j \neq 1}}^{n} \widetilde{c}_{i j}\right\} \\
& \leq h_{i}, \quad i=1, \ldots, n,
\end{aligned}
$$

which actually means

$$
\mathbf{g}(\mathbf{h}) \leq \dot{\mathbf{h}}, \quad t \in\left[t_{0}, T\right) .
$$

In other words, the vector function $\mathbf{h}(t)$, we have considered above, should be an arbitrary PS $\mathbf{y}(t)>0$ of DI (2.19). Hence,

$$
0<\mathbf{z}(t) \leq \mathbf{y}(t), \quad t \in\left[t_{0}, T\right)
$$

for all PSs $\mathbf{z}(t)=\mathbf{z}\left(t ; t_{0}, \mathbf{z}_{0}\right)$ of DE (3.1), corresponding to initial conditions that satisfy the inequality

$$
0<\mathbf{z}\left(t_{0}\right)=\mathbf{z}_{0} \leq \mathbf{y}\left(t_{0}\right)
$$

The PS solution $\mathbf{z}^{*}(t)=\mathbf{z}\left(t, t_{0}, \mathbf{y}\left(t_{0}\right)\right)$ of DE (3.1), which corresponds to the initial condition $\mathbf{y}\left(t_{0}\right)>0$, satisfies the inequality

$$
0<\mathbf{z}^{*}(t) \leq \mathbf{y}(t), \quad t \in\left[t_{0}, T\right),
$$

but, at the same time, it is one of the PSs of DI (2.19) with the initial condition $\mathbf{y}\left(t_{0}\right)>0$, and, consequently, it is able to ensure

$$
0<\mathbf{z}(t) \leq \mathbf{z}^{*}(t), \quad t \in\left[t_{0}, T\right) .
$$

THEOREM 3.4. If $\mathbf{H}_{\mathbf{y}}(t), \mathbf{H}_{\mathbf{z} *}(t)$, and $\mathbf{H}_{\mathbf{z}}(t)$ denote three TDRSs, which are FI with respect to UNS (2.1), generated by the following three types of PSs of DI 
(2.19), $\mathbf{y}(t)$-arbitrary PS of DI (2.19), $\mathbf{z}^{*}(t)$-unique PS of DE (3.1), with $\mathbf{z}^{*}\left(t_{0}\right)=$ $\mathbf{y}\left(t_{0}\right)$, and $\mathbf{z}(t)$-arbitrary PS of DE (3.1), with $\mathbf{z}\left(t_{0}\right) \leq \mathbf{y}\left(t_{0}\right)$, then

$$
\mathbf{H}_{\mathbf{z}}(t) \subseteq \mathbf{H}_{\mathbf{z} *}(t) \subseteq \mathbf{H}_{\mathbf{y}}(t) \quad \forall t \in\left[t_{0}, T\right),
$$

where $\left[t_{0}, T\right)$ denotes the maximal interval of existence for $\mathbf{H}_{\mathbf{y}}(t)$.

PROOF. The construction procedure of $\mathbf{H}_{\mathbf{y}}(t), \mathbf{H}_{\mathbf{z} *}(t)$, and $\mathbf{H}_{\mathbf{z}}(t)$ guarantees, according to Lemma 3.3, the following inclusions:

$$
\left[-z_{i}(t), z_{i}(t)\right] \subseteq\left[-z_{i}^{*}(t), z_{i}^{*}(t)\right] \subseteq\left[-y_{i}(t), y_{i}(t)\right], \quad t \in\left[t_{0}, T\right), i=1, \ldots, n
$$

Now, taking the Cartesian product (2.3) that defines the TDRSs, we complete the proof.

Given a TDRS which is FI with respect to UNS (2.1), we can formulate a condition for the existence of other TDRSs, strictly included in the former one, which are FI with respect to UNS (2.1) too.

THEOREM 3.5. Denote by $\mathbf{H}_{\mathbf{y}}(t)$ a TDRS, which is FI with respect to UNS (2.1) for its maximal interval of existence $\left[t_{0}, T\right)$. If there exist $n$ functions $\delta_{i}(t) \in \mathbb{C}^{1}$, which are nondecreasing, positive, and subunitary $0<\delta_{i}(t)<1, i=1, \ldots, n$, such that

$$
\mathbf{g}(\Delta(t) \mathbf{y}(t)) \leq \Delta(t) \mathbf{g}(\mathbf{y}(t)) ; \quad \Delta(t)=\operatorname{diag}\left\{\delta_{i}(t), \ldots, \delta_{n}(t)\right\}
$$

then the TDRS $\mathbf{H}_{\Delta \mathbf{y}}(t)$, generated by the vector function $\Delta(t) \mathbf{y}(t)$, is also FI with respect to UNS (2.1) and

$$
\mathbf{H}_{\Delta \mathbf{y}}(t) \subset \mathbf{H}_{\mathbf{y}}(t), \quad t \in\left[t_{0}, T\right) .
$$

Proof. As $\mathbf{H}_{\mathbf{y}}(t)$ is FI with respect to UNS (2.1), the vector function $\mathbf{y}(t)$ is a PS of DI (2.19), and, consequently, the following inequality holds for $t \in\left[t_{0}, T\right)$ :

$$
\Delta(t) \mathbf{g}(\mathbf{y}(t)) \leq \Delta(t) \dot{\mathbf{y}}(t)
$$

due to the positiveness of $\delta_{i}(t), i=1, \ldots, n$.

Taking into account the monotonicity of $\delta_{i}(t), i=1, \ldots, n$, and the positiveness of $\mathbf{y}(t)$, we can also write

$$
\Delta(t) \mathbf{g}(\mathbf{y}(t)) \leq \Delta(t) \dot{\mathbf{y}}(t)+\dot{\Delta}(t) \mathbf{y}(t)=\frac{d}{d t}(\Delta(t) \mathbf{y}(t)), \quad t \in\left[t_{0}, T\right),
$$

which is further exploited, together with inequality (3.48), to show that $\Delta(t) \mathbf{y}(t)$ is a PS of DI (2.19). Hence, TDRS $\mathbf{H}_{\Delta \mathbf{y}}(t)$ is FI with respect to UNS (2.1). 
On the other hand, the conditions $\delta_{i}(t)<1, i=1, \ldots, n$, imply the strict inclusions

$$
\left[-\delta_{i}(t) y_{i}(t), \delta_{i}(t) y_{i}(t)\right] \subset\left[-y_{i}(t), y_{i}(t)\right], \quad i=1, \ldots, n, t \in\left[t_{0}, T\right),
$$

which, in accordance with the definition of TDRSs in (2.3), complete the proof.

REMARK 3.6. Functions $\delta_{i}(t)$ can be chosen as positive, subunitary constants, a case in which the resulting TDRS $\mathbf{H}_{\Delta \mathbf{y}}(t)$ is homotetic with $\mathbf{H}_{\mathbf{y}}(t)$, taking different transformation factors for each component. When all $\delta_{i}, i=$ $1, \ldots, n$, are equal to the same positive, subunitary constant, the transformation factors are identical for all the components.

A great interest for practice presents those TDRSs, FI with respect to UNS (2.1), which are defined on $\left[t_{0}, \infty\right)$ and remain bounded for any $t \in\left[t_{0}, \infty\right)$. Therefore, our next theorem deals with the case of infinite-time horizon, for which it gives a necessary condition formulated directly in terms of intervaltype coefficients $a_{i i}$ and exponents $p_{i i}$ in UNS (2.1).

THEOREM 3.7. For the existence of TDRSs, FI with respect to UNS (2.1), which are bounded on $\left[t_{0}, \infty\right)$, it is necessary (but not sufficient) that $a_{i i}$ and $p_{i i}$ of UNS (2.1) meet the following requirement, for $i=1, \ldots, n$ :

$$
\text { (if } \left.p_{i i} \text { odd, then } a_{i i}^{+} \leq 0\right) \quad \text { or } \quad\left(\text { if } p_{i i} \text { even, then } a_{i i}^{-}=a_{i i}^{+}=0\right) \text {. }
$$

Proof. The boundedness on $\left[t_{0}, \infty\right)$ of a TDRS $\mathbf{H}_{\mathbf{y}}(t)$ which is FI with respect to UNS (2.1) means, according to Theorem 2.4, the existence of PSs for DI (2.19) which are bounded on $\left[t_{0}, \infty\right)$. Denote by $\mathbf{y}(t)$ such a solution of DI (2.19). Denote by $\mathbf{z}^{*}(t)$ the PS of DE (3.1) which corresponds to the initial condition $\mathbf{z}^{*}\left(t_{0}\right)=\mathbf{y}\left(t_{0}\right)>0$. From Lemma 3.3, it follows that $\mathbf{z}^{*}(t)$ is also bounded.

On the other hand, the second part of the proof of Lemma 3.2 shows that $z_{i}^{*}(t), i=1, \ldots, n$, should satisfy the inequality

$$
\dot{z}_{i}^{*}(t) \geq \hat{c}_{i i}\left(z_{i}^{*}(t)\right)^{p_{i i}}, \quad \hat{c}_{i i}=\max \left\{\bar{c}_{i i}, \widetilde{c}_{i i}\right\}
$$

If, for arbitrary $i, i=1, \ldots, n$, we attach the differential equation

$$
\dot{r}_{i}(t)=\hat{c}_{i i} r_{i}^{p_{i i}}(t)
$$

with the initial condition

$$
r_{i}\left(t_{0}\right)=z_{i}^{*}\left(t_{0}\right), \quad i=1, \ldots, n,
$$


we can use the link between $z_{i}^{*}(t)$ and $r_{i}(t)$ (e.g., [6, page 57]). Consequently, for the maximal interval of existence of both $z_{i}^{*}(t)$ and $r_{i}(t)$, we get

$$
z_{i}^{*}(t) \geq r_{i}(t)
$$

which, corroborated with the boundedness requirement for $z_{i}^{*}(t)$, implies the boundedness of $r_{i}(t)$ on $\left[t_{0}, T\right)$.

For the solutions $r_{i}(t)$, we have the following analytical expressions:

(i) if $\hat{c}_{i i}=0, r_{i}(t)=r_{i}\left(t_{0}\right)$,

(ii) if $\hat{c}_{i i} \neq 0$ and $p_{i i}=1, r_{i}(t)=r_{i}\left(t_{0}\right) e^{\hat{c}_{i i}\left(t-t_{0}\right)}$,

(iii) if $\hat{c}_{i i} \neq 0$ and $p_{i i} \geq 2, r_{i}(t)=1 / \sqrt[p_{i i}-1]{-\hat{c}_{i i}\left(p_{i i}-1\right)\left(t-t_{0}\right)+1 / r_{i}^{p_{i i}-1}\left(t_{0}\right)}$, showing that, for the boundedness of $r_{i}(t)$ on $\left[t_{0}, \infty\right)$, we need $\hat{c}_{i i} \leq 0$.

Now, taking into account formulation (2.7) and (2.8) of $\bar{c}_{i i}, \tilde{c}_{i i}$ in terms of the interval type coefficients $a_{i i}$ of UNS (2.1), we see that there exist the following possibilities:

(i) if $p_{i i}$ odd, then, with necessity, $a_{i i}^{+} \leq 0$,

(ii) if $p_{i i}$ even, then, with necessity, $a_{i i}^{-}=a_{i i}^{+}=0$.

The proof is completed because the previous discussion is valid for any $i$, $i=1, \ldots, n$.

The next step in refining the conditions imposed on the TDRS FI with respect to UNS (2.1) aims at forcing the boundedness property by adding a supplementary request for the time-dependence of TDRS, namely, to approach $\{0\}$ for $t \rightarrow \infty$. Thus, the concept of FI induces a particular type of asymptotic stability (AS) for the equilibrium point (EP) $\{0\}$ of UNS (2.1) (stronger than the standard concept based on vector norms in $\mathbb{R}^{n}$ ), which is going to be separately studied in the following section.

4. Componentwise asymptotic stability. Let $\boldsymbol{\gamma}(t):\left[t_{0}, \infty\right) \rightarrow \mathbb{R}^{n}$ be a differentiable vector function with $\gamma_{i}(t)>0, i=1, \ldots, n$, (as considered in Definition 2.1, which introduces the FI concept), and suppose that $\boldsymbol{\gamma}(t)$ also has the property

$$
\lim _{t \rightarrow \infty} \boldsymbol{\gamma}(t)=0
$$

DEFINITION 4.1. EP $\{0\}$ of UNS (2.1) is called componentwise asymptotically stable with respect to $\boldsymbol{\gamma}(t)\left(\mathrm{CWAS}_{\boldsymbol{\gamma}}\right)$ if, for any $\mathbf{x}\left(t_{0}\right)=\mathbf{x}_{0}$ with $\left|\mathbf{x}_{0}\right| \leq \boldsymbol{\gamma}\left(t_{0}\right)$, the following inequality holds:

$$
|\mathbf{x}(t)| \leq \boldsymbol{\gamma}(t), \quad t \in\left[t_{0}, \infty\right) .
$$

REMARK 4.2. Definition 4.1 can be restated in terms of FI by taking Definition 2.1 with $T=\infty$ in (2.4) supplemented with condition (4.1) for the behavior at the infinity. 
In the light of the above remark, the results presented in Theorems 2.3 and 2.4 can be immediately transformed to characterize $\mathrm{CWAS}_{\gamma}$ of EP\{0\} of UNS (2.1), yielding the following two theorems.

THEOREM 4.3. P\{0\} of UNS (2.1) is $\mathrm{CWAS}_{\gamma}$ if and only if there exist common PSs $\boldsymbol{\gamma}(t)>0$ for DI (2.18), with $\lim _{t \rightarrow \infty} \boldsymbol{\gamma}(t)=0$.

Proof. It is a direct consequence of Theorem 2.3 for the particular case of TDRSs meeting condition (4.1).

THEOREM 4.4. EP\{0\} of UNS (2.1) is $\mathrm{CWAS}_{\gamma}$ if and only if there exist PSs $\boldsymbol{\gamma}(t)>0$ for DI (2.19), with $\lim _{t \rightarrow \infty} \boldsymbol{\gamma}(t)=0$.

Proof. It is a direct consequence of Theorem 2.4 for the particular case of TDRSs meeting condition (4.1).

As the boundedness of TDRSs on $\left[t_{0}, \infty\right)$ introduces some restrictions for the exponents $p_{i i}$ and interval-type coefficients $a_{i i}$ of UNS (2.1) (formulated in Theorem 3.7), more restrictive conditions are expected when replacing boundedness with the stronger requirement (4.1).

THEOREM 4.5. A necessary condition for EP\{0\} of UNS (2.1) to be $\mathrm{CWAS}_{y}$ is that $p_{i i}$ odd and $a_{i i}^{+}<0$ for all $i=1, \ldots, n$.

Proof. From the fact that EP $\{0\}$ of UNS (2.1) is $\mathrm{CWAS}_{\gamma}$, it follows that, for each $i, i=1, \ldots, n$, there exists a number of points where $\gamma_{i}(t)$ is decreasing. Let $t^{*}$ be one of them, meaning that $\dot{\gamma}_{i}\left(t^{*}\right)<0$. Taking into account that $\boldsymbol{\gamma}(t)$ is a PS of DI (2.18), we get the inequalities

$$
\begin{aligned}
& 0>\dot{\gamma}_{i}\left(t^{*}\right) \geq \bar{g}_{i}\left(\dot{\gamma}_{i}\left(t^{*}\right)\right) \geq \bar{c}_{i i} \gamma_{i}\left(t^{*}\right), \\
& 0>\dot{\gamma}_{i}\left(t^{*}\right) \geq \tilde{g}_{i}\left(\dot{\gamma}_{i}\left(t^{*}\right)\right) \geq \tilde{c}_{i i} \gamma_{i}\left(t^{*}\right),
\end{aligned}
$$

which, because of the positiveness of $\boldsymbol{\gamma}\left(t^{*}\right)$, yield the necessary condition

$$
\hat{c}_{i i}=\max \left\{\bar{c}_{i i}, \tilde{c}_{i i}\right\}<0 .
$$

The proof continues along the lines of the proof of Theorem 3.7 in order to restate this condition in terms of $p_{i i}$ and $a_{i i}, i=1, \ldots, n$, of the considered UNS (2.1).

We now resume our qualitative analysis of the solutions of DI (2.19) and DE (3.1) to develop a refined interpretation of the result stated in Theorem 4.4.

LEMMA 4.6. Let $p_{i i}$ odd and $a_{i i}^{+}<0$ for all $i=1, \ldots, n$. Consider an arbitrary PS $\mathbf{y}(t)>0$ of DI (2.19) with its maximal interval of existence $\left[t_{0}, T\right)$. If $\mathbf{z}(t)$ denotes an arbitrary solution of DE (3.1), corresponding to the initial condition $\mathbf{z}\left(t_{0}\right)$, satisfying

$$
-\mathbf{y}\left(t_{0}\right) \leq \mathbf{z}\left(t_{0}\right) \leq \mathbf{y}\left(t_{0}\right)
$$


then the following inequalities hold for $t \in\left[t_{0}, T\right)$ :

$$
-\mathbf{y}(t) \leq \mathbf{z}(t) \leq \mathbf{y}(t)
$$

Proof. Suppose that there exists a vector function $\mathbf{h}(t) \in \mathbb{R}^{n}$, differentiable and positive for $t \in\left[t_{0}, T\right)$, with the following property: any solution of DE (3.1) $\mathbf{z}(t)=\mathbf{z}\left(t ; t_{0}, z_{0}\right)$, whose initial condition satisfies the inequality

$$
-\mathbf{h}\left(t_{0}\right) \leq \mathbf{z}\left(t_{0}\right) \leq \mathbf{h}\left(t_{0}\right)
$$

satisfies the inequality

$$
-\mathbf{h}(t) \leq \mathbf{z}(t) \leq \mathbf{h}(t), \quad t \in\left[t_{0}, T\right) .
$$

According to [11, pages 74-75], a necessary and sufficient condition for such a property to take place is that

$$
\begin{aligned}
g_{i}\left(z_{1}, \ldots, z_{i-1}, h_{i}, z_{i+1}, \ldots, z_{n}\right) & \leq \dot{h}_{i}, \quad i=1, \ldots, n, \\
g_{i}\left(z_{1}, \ldots, z_{i-1},-h_{i}, z_{i+1}, \ldots, z_{n}\right) & \geq-\dot{h}_{i}, \quad i=1, \ldots, n,
\end{aligned}
$$

for all $\mathbf{z}(t)$, with $-\mathbf{h}(t) \leq \mathbf{z}(t) \leq \mathbf{h}(t)$. Using the definition of $g_{i}$ in (2.19), we can write

$$
\begin{aligned}
g_{i}\left(z_{1}, \ldots, z_{i-1}, h_{i}, z_{i+1}, \ldots, z_{n}\right) & \\
= & \max _{h_{i}, z_{j}, j \neq i}\left\{\bar{c}_{i i} h_{i}^{p_{i i}}+\sum_{\substack{j=1 \\
j \neq i}}^{n} \bar{c}_{i j} z_{j}^{p_{i j}}, \widetilde{c}_{i i} h_{i}^{p_{i i}}+\sum_{\substack{j=1 \\
j \neq i}}^{n} \widetilde{c}_{i j} z_{j}^{p_{i j}}\right\} \\
& \leq \max _{\mathbf{h} \in \mathbb{R}^{n}}\left\{\bar{c}_{i i} h_{i}^{p_{i i}}+\sum_{\substack{j=1 \\
j \neq i}}^{n} \bar{c}_{i j} h_{j}^{p_{i j}}, \widetilde{c}_{i i} h_{i}^{p_{i i}}+\sum_{\substack{j=1 \\
j \neq i}}^{n} \widetilde{c}_{i j} h_{j}^{p_{i j}}\right\} \\
& \leq \dot{h}_{i}, \quad i=1, \ldots, n,
\end{aligned}
$$

which actually means DI (2.19) (written in $\mathbf{h}$ instead of $\mathbf{y})$ and

$$
\begin{aligned}
& g_{i}\left(z_{1}, \ldots, z_{i-1},-h_{i}, z_{i+1}, \ldots, z_{n}\right) \\
& \quad=\max _{h_{i}, z_{j}, j \neq i}\left\{\bar{c}_{i i}\left(-h_{i}\right)^{p_{i i}}+\sum_{\substack{j=1 \\
\neq i}}^{n} \bar{c}_{i j} z_{j}^{p_{i j}}, \widetilde{c}_{i i}\left(-h_{i}\right)^{p_{i i}}+\sum_{\substack{j=1 \\
j \neq i}}^{n} \tilde{c}_{i j} z_{j}^{p_{i j}}\right\} \\
& \quad \geq \sum_{\substack{j=1 \\
p_{i j} \text { odd }}}^{n} c_{i j}\left(-h_{j}\right)^{p_{i j}} \geq-\dot{h}_{i}, \quad i=1, \ldots, n,
\end{aligned}
$$

where $c_{i j}=\bar{c}_{i j}=\tilde{c}_{i j}$ because all $p_{i j}$ are odd. 
The last set of inequalities means

$$
\dot{h}_{i} \geq \sum_{\substack{j=1 \\ p_{i j} \text { odd }}}^{n} c_{i j}\left(h_{j}\right)^{p_{i j}}, \quad i=1, \ldots, n
$$

which is automatically satisfied whenever DI (2.19) is met because each inequality in DI (2.19) also includes a positive amount given by the sum corresponding to the even $p_{i j}$.

Thus, we have shown that the vector function $\mathbf{h}(t)$, considered above, should be a PS of DI (2.19), a fact that completes the proof.

Lemma 4.6 creates a deeper insight into the topology of the solutions (not only positive) of DE (3.1) in the vicinity of $\mathrm{EP}\{0\}$, which permits revealing the link between condition (4.1) and the nature of $\mathrm{EP}\{0\}$ for DE (3.1).

THEOREM 4.7. EP\{0\} of UNS (2.1) is $\mathrm{CWAS}_{\gamma}$ if and only if EP\{0\} of DE (3.1) is AS.

\section{ProOF}

SuFFICIENCY. The statement "EP\{0\} of DE (3.1) is AS" ensures the existence of a vicinity of $\{0\}$, denoted by $V(0)$, such that, for any $\mathbf{z}\left(t_{0}\right)=\mathbf{z}_{0} \in V(0)$, the solution $\mathbf{z}(t)=\mathbf{z}\left(t ; t_{0}, z_{0}\right)$ of DE (3.1) has the property $\lim _{t \rightarrow \infty} \mathbf{z}(t)=0$. If, in $V(0)$, we take a positive initial condition $\mathbf{z}^{*}\left(t_{0}\right)=\mathbf{z}_{0}^{*}>0$, then, in accordance with Lemma 3.2, the corresponding solution $\mathbf{z}^{*}(t)$ of DE (3.1) will remain positive on $\left[t_{0}, \infty\right)$ with $\lim _{t \rightarrow \infty} \mathbf{z}^{*}(t)=0$. Thus, we have found a PS for DI (2.19), which also meets condition (4.1); from Theorem 4.4, it results that $\operatorname{EP}\{0\}$ of UNS (2.1) is $\mathrm{CWAS}_{\boldsymbol{\gamma}}$ with $\boldsymbol{\gamma}(t)=\mathbf{z}^{*}(t)$.

NECESSITY. The statement "EP\{0\} of UNS (2.1) is CWAS ${ }_{\gamma}$ " ensures the existence of a PS $\boldsymbol{\gamma}(t)$ for DI (2.19), defined on $\left[t_{0}, \infty\right)$ and with $\lim _{t \rightarrow \infty} \boldsymbol{\gamma}(t)=0$. According to Lemma 4.6, this means that, for any $\mathbf{z}\left(t_{0}\right)=\mathbf{z}_{0}$ with $-\boldsymbol{\gamma}\left(t_{0}\right) \leq$ $\mathbf{z}\left(t_{0}\right) \leq \boldsymbol{\gamma}(t)$, the corresponding solution $\mathbf{z}(t)=\mathbf{z}\left(t ; t_{0}, z_{0}\right)$ of DE (3.1) has the property

$$
-\boldsymbol{\gamma}(t) \leq \mathbf{z}(t) \leq \boldsymbol{\gamma}(t), \quad t \in\left[t_{0}, \infty\right),
$$

with $\lim _{t \rightarrow \infty} \boldsymbol{\gamma}(t)=0$. It results that $\mathrm{EP}\{0\}$ of DE (3.1) is AS.

REMARK 4.8. Clearly, the concept of $\mathrm{CWAS}_{y}$ for EP\{0\} of UNS (2.1) is not equivalent to the standard AS. If $\mathrm{EP}\{0\}$ of UNS (2.1) is $\mathrm{CWAS}_{\boldsymbol{\gamma}}$, then it is AS, but the converse statement is not true. However, Theorem 4.7 can be used as a sufficient condition for approaching the standard problem of AS for UNS (2.1), where the presence of uncertainties (expressed by interval-type coefficients) makes rather difficult the usage of classical procedures. 
For practice, it is hard to handle DE (3.1) in order to check its AS analytically. A more attractive approach is to find just a sufficient condition for $\mathrm{CWAS}_{\boldsymbol{\gamma}}$, based on an operator with a more tractable form than $\mathbf{g}$ in DE (3.1).

THEOREM 4.9. Consider the DE

$$
\dot{\mathbf{z}}=\hat{\mathbf{g}}(\mathbf{z}) ; \quad \hat{g}_{i}(\mathbf{z})=\sum_{j=1}^{n} \hat{c}_{i j} z_{j}^{p_{i j}}, \quad i=1, \ldots, n,
$$

where the coefficients $\hat{c}_{i j}$ are defined by

$$
\hat{c}_{i j}=\max \left\{\bar{c}_{i j}, \widetilde{c}_{i j}\right\}, \quad i, j=1, \ldots, n
$$

(i) If $E P\{0\}$ is AS for DE (4.14), then $E P\{0\}$ is $\mathrm{CWAS}_{y}$ for UNS (2.1).

(ii) In the particular case when the interval-type coefficients $a_{i j}$ of UNS (2.1) satisfy the inequalities given below, for each $i, i=1, \ldots, n$,

$$
\text { if } p_{i j j} \text { even then }\left(a_{i j}^{+} \geq-a_{i j}^{-} \forall j \text { or } a_{i j}^{+} \leq-a_{i j}^{-} \forall j\right) \text {, }
$$

the sufficient condition stated at (i) is also necessary for EP\{0\} of UNS (2.1) to be $\mathrm{CWAS}_{\gamma}$.

Proof. (i) We can easily see that the techniques used in the proof of Lemma 3.2 can be immediately applied to show that, for any positive initial condition $\mathbf{y}\left(t_{0}\right)=\mathbf{y}_{0}>0$, the corresponding solution $\mathbf{y}(t)$ of $\mathrm{DE}$ (4.14) remains positive as long as it exists. Thus, from the AS of EP\{0\} of DE (4.14), it results that $\mathrm{DE}$ (4.14) has PSs, generically denoted by $\mathbf{y}(t)$ defined on $\left[t_{0}, \infty\right)$ and meeting condition (4.1).

On the other hand, the following inequality

$$
\hat{\mathbf{g}}(\mathbf{y}) \geq \mathbf{g}(\mathbf{y})
$$

holds for all $\mathbf{y} \in \mathbb{R}_{+}^{n}$. Finally, we see that, for any PS of DE (4.14) with the properties mentioned above, we can write

$$
\dot{\mathbf{y}}=\hat{\mathbf{f}}(\mathbf{y}) \geq \mathbf{g}(\mathbf{y})
$$

This means that $\mathbf{y}(t)>0$ is a PS for DI (2.19), also meeting condition (4.1), that is, UNS (2.1) is $\mathrm{CWAS}_{\boldsymbol{\gamma}}$, with $\boldsymbol{\gamma}(t)=\mathbf{y}(t)$.

(ii) A quick exploration of the definition of $\hat{\mathbf{g}}$ in (4.14) shows that the difference from $\mathbf{g}$ in (2.19) actually refers to the sums corresponding to $p_{i j}$ even, 
that is,

$$
\begin{gathered}
\sum_{\substack{j=1 \\
j \neq i \\
p_{i j} \text { even }}}^{n} \hat{c}_{i j} z_{j}^{p_{i j}} \quad \text { which appears in } \hat{g}_{i} ; \\
\sum_{\substack{j=1 \\
j \neq i \\
p_{i j} \text { even }}}^{n} \bar{c}_{i j} z_{j}^{p_{i j}} \quad \text { which appears in } \overline{\mathfrak{g}}_{i} ; \\
\sum_{\substack{j=1 \\
j \neq i \\
p_{i j} \text { even }}}^{n} \tilde{c}_{i j} z_{j}^{p_{i j}} \quad \text { which appears in } \tilde{g}_{i} ; \quad i=1, \ldots, n .
\end{gathered}
$$

Condition (4.16), expressed in terms of $\bar{c}_{i j}$ and $\tilde{c}_{i j}$, means, for each $i=$ $1, \ldots, n$

(a) $\bar{c}_{i j} \geq \tilde{c}_{i j}$ for all $j$ corresponding to $p_{i j}$ even, or

(b) $\tilde{c}_{i j} \geq \bar{c}_{i j}$ for all $j$ corresponding to $p_{i j}$ even.

The inequalities in case (a) show that

$$
\overline{\mathfrak{g}}_{i}(\mathbf{z}) \geq \tilde{\mathfrak{g}}_{i}(\mathbf{z}), \quad \mathbf{z} \in \mathbb{R}^{n},
$$

yielding the conclusion (because $p_{i j}$ are even)

$$
\mathcal{g}_{i}(\mathbf{z})=\max _{\mathbf{z}}\left\{\bar{g}_{i}(\mathbf{z}), \tilde{\mathfrak{g}}_{i}(\mathbf{z})\right\}=\overline{\mathfrak{g}}_{i}(\mathbf{z})=\hat{\mathfrak{g}}_{i}(\mathbf{z}), \quad \mathbf{z} \in \mathbb{R}^{n}
$$

The inequalities in case (b) show that

$$
\tilde{\mathfrak{g}}_{i}(\mathbf{z}) \geq \overline{\mathfrak{g}}_{i}(\mathbf{z}), \quad \mathbf{z} \in \mathbb{R}^{n},
$$

yielding the conclusion (because $p_{i j}$ are even)

$$
\mathcal{g}_{i}(\mathbf{z})=\max _{\mathbf{z}}\left\{\overline{\mathfrak{g}}_{i}(\mathbf{z}), \tilde{\mathfrak{g}}_{i}(\mathbf{z})\right\}=\tilde{\mathfrak{g}}_{i}(\mathbf{z})=\hat{\mathfrak{g}}_{i}(\mathbf{z}), \quad \mathbf{z} \in \mathbb{R}^{n}
$$

Thus, taking all $i=1, \ldots, n$, we can write

$$
\mathbf{g}(\mathbf{z})=\hat{\mathbf{g}}(\mathbf{z}), \quad \mathbf{z} \in \mathbb{R}^{n}
$$

This equality allows the replacement of $\mathbf{g}(\mathbf{z})$ by $\hat{\mathbf{g}}(\mathbf{z})$ in Theorem 4.7, which is a necessary and sufficient condition.

The advantage of Theorem 4.9 consists in dealing with a single DE (4.14) whose coefficients have unique and constant values. Moreover, inequalities (4.16) may be frequently fulfilled in practical studies, a fact that ensures a complete answer to the $\mathrm{CWAS}_{\boldsymbol{\gamma}}$ investigation. 
5. Componentwise exponential asymptotic stability. Consider the vector function

$$
\boldsymbol{\gamma}(t)=\boldsymbol{\alpha} e^{r t}, \quad \boldsymbol{\alpha}=\left[\alpha_{1}, \ldots, \alpha_{n}\right]^{\prime} \in \mathbb{R}^{n}, \quad \alpha_{i}>0, i=1, \ldots, n, r<0
$$

DEFINITION 5.1. EP $\{0\}$ of UNS (2.1) is called componentwise exponential asymptotically stable (CWEAS) if there exist $\boldsymbol{\alpha} \in \mathbb{R}^{n}, \boldsymbol{\alpha}>0$, and $r<0$ such that, for any $\mathbf{x}\left(t_{0}\right)=\mathbf{x}_{0}$ with $\left|\mathbf{x}_{0}\right| \leq \boldsymbol{\alpha} e^{r t_{0}}$, the following inequality holds

$$
|\mathbf{x}(t)| \leq \boldsymbol{\alpha} e^{r t}, \quad t \in\left[t_{0}, \infty\right)
$$

REMARK 5.2. Definition 5.1 can be restated in terms of Definition 4.1 by taking for $\boldsymbol{\gamma}(t)$ the particular form given by (5.1).

It is natural to understand this particularization of the function $\boldsymbol{\gamma}(t)$ as a new restriction on the time-dependence of TDRS (2.3), which imposes a certain decreasing rate that should be followed by the state trajectories of UNS (2.1). Such a restriction is reflected by more severe conditions on the exponents $p_{i i}$ and interval-type coefficients $a_{i i}$ of UNS (2.1) than stated in Theorem 4.5.

THEOREM 5.3. A necessary condition for the EP\{0\} of UNS (2.1) to be CWEAS is that $p_{i i}=1$ and $a_{i i}^{+}<0, i=1, \ldots, n$, in UNS (2.1).

Proof. If EP $\{0\}$ of UNS (2.1) is CWEAS, then, according to Theorem 4.3 and Remark 5.2, there exist $\boldsymbol{\alpha} \in \mathbb{R}^{n}, \boldsymbol{\alpha}>0$, and $r<0$ such that

$$
\begin{aligned}
0>\alpha_{i} r e^{r t} \geq \bar{g}_{i}\left(\boldsymbol{\alpha} e^{r t}\right) & =\bar{c}_{i i} \alpha_{i}^{p_{i i}} e^{p_{i i} r t} \sum_{\substack{j=1 \\
j \neq i}}^{n} c_{i j} \alpha_{j}^{p_{i j}} e^{p_{i i} r t} \\
& \geq \bar{c}_{i i} \alpha_{i}^{p_{i i}} e^{p_{i i} r t}, \quad t \in\left[t_{0}, \infty\right), i=1, \ldots, n, \\
0>\alpha_{i} r e^{r t} \geq \tilde{g}_{i}\left(\boldsymbol{\alpha} e^{r t}\right) & =\tilde{c}_{i i} \alpha_{i}^{p_{i i}} e^{p_{i i} r t}+\sum_{\substack{j=1 \\
j \neq i}}^{n} c_{i j} \alpha_{j}^{p_{i j}} e^{p_{i i} r t} \\
& \geq \widetilde{c}_{i i} \alpha_{i}^{p_{i i}} e^{p_{i i} r t}, \quad t \in\left[t_{0}, \infty\right), i=1, \ldots, n .
\end{aligned}
$$

It follows that we necessarily have

$$
\bar{c}_{i i}<0, \quad \tilde{c}_{i i}<0, \quad p_{i i} r=r,
$$

which, in terms of UNS (2.1), means $a_{i i}^{+}<0$ and $p_{i i}=1$. 
THEOREM 5.4. EP\{0\} of UNS (2.1) is CWEAS if and only if the following nonlinear algebraic inequalities are compatible (have solutions $\alpha_{i}>0, i=1, \ldots, n$, $r<0)$ :

$$
\begin{aligned}
& \sum_{j=1}^{n} \frac{\bar{c}_{i j} \alpha_{i}^{p_{i j}}}{\alpha_{i}} \leq r ; \quad i=1, \ldots, n, \\
& \sum_{j=1}^{n} \frac{\widetilde{c}_{i j} \alpha_{j}^{p_{i j}}}{\alpha_{i}} \leq r ; \quad i=1, \ldots, n .
\end{aligned}
$$

Proof. The statement "EP\{0\} of UNS (2.1) is CWEAS" is equivalent with the existence of $\alpha_{i}>0, i=1, \ldots, n$, and $r<0$ such that

$$
\begin{aligned}
& \sum_{j=1}^{n} \bar{c}_{i j} \alpha_{j}^{p_{i j}} e^{p_{i j} r t} \leq \alpha_{i} r e^{r t}, \quad t \in\left[t_{0}, \infty\right), i=1, \ldots, n, \\
& \sum_{j=1}^{n} \tilde{c}_{i j} \alpha_{j}^{p_{i j}} e^{p_{i j} r t} \leq \alpha_{i} r e^{r t}, \quad t \in\left[t_{0}, \infty\right), i=1, \ldots, n .
\end{aligned}
$$

The necessity of (5.5) results from the above inequalities by taking $t=0$. For the sufficiency of (5.5), we take into account that $p_{i i}=1, i=1, \ldots, n$, (according to Theorem 5.3), and for arbitrary $t \in\left[t_{0}, \infty\right)$, we can write

$$
\begin{aligned}
\sum_{j=1}^{n} \bar{c}_{i j} e^{p_{i j} r t} \alpha_{j}^{p_{i j}} & =\bar{c}_{i i} e^{r t} \alpha_{i}+\sum_{\substack{j=1 \\
j \neq i}}^{n} \bar{c}_{i j} e^{p_{i j} r t} \alpha_{j}^{p_{i j}} \leq \bar{c}_{i i} e^{r t} \alpha_{i}+\sum_{\substack{j=1 \\
j \neq i}}^{n} \bar{c}_{i j} e^{r t} \alpha_{j}^{p_{i j}} \\
& =\left(\sum_{\substack{j=1 \\
j \neq i}}^{n} \bar{c}_{i j} \alpha_{j}^{p_{i j}}\right) e^{r t} \leq\left(\alpha_{i} r\right) e^{r t}=\alpha_{i} r e^{r t}, \quad i=1, \ldots, n,
\end{aligned}
$$

and, similarly,

$$
\sum_{j=1}^{n} \widetilde{c}_{i j} e^{p_{i j} r t} \alpha_{j}^{p_{i j}} \leq \alpha_{i} r e^{r t}, \quad i=1, \ldots, n .
$$

THEOREM 5.5. EP\{0\} of UNS (2.1) is CWEAS if and only if the following nonlinear algebraic inequalities are compatible (have solutions $\alpha_{i}>0, i=1, \ldots, n$ ):

$$
\begin{aligned}
& \sum_{j=1}^{n} \frac{\bar{c}_{i j} \alpha_{i}^{p_{i j}}}{\alpha_{i}}<0 ; \quad i=1, \ldots, n, \\
& \sum_{j=1}^{n} \frac{\widetilde{c}_{i j} \alpha_{j}^{p_{i j}}}{\alpha_{i}}<0 ; \quad i=1, \ldots, n .
\end{aligned}
$$

Proof. It is an immediate consequence of Theorem 5.4 due to negativity of $r$. 
Conditioning the existence of CWEAS to the values of $p_{i i}$ in UNS (2.1) (as stated in Theorem 5.3) raises a direct question about the link between CWEAS and $\mathrm{CWAS}_{\boldsymbol{\gamma}}$.

THEOREM 5.6. For $p_{i i}=1$ and $a_{i i}^{+}<0, i=1, \ldots, n$, the EP\{0\} of UNS (2.1) is $\mathrm{CWAS}_{\gamma}$ if and only if it is CWEAS.

\section{PROOF}

SUFFICIENCY. This is obvious according to Definition 5.1 and Remark 5.2.

NeCESSITY. According to Theorem 5.5, when multiplying each inequality (5.10) and (5.11) by $\alpha_{i}>0, i=1, \ldots, n$, we get that the CWEAS condition for $\mathrm{EP}\{0\}$ of UNS (2.1) is equivalent to

$$
\overline{\mathbf{g}}(\boldsymbol{\alpha})<0, \quad \tilde{\mathbf{g}}(\boldsymbol{\alpha})<0,
$$

where $\overline{\mathbf{g}}$ and $\tilde{\mathbf{g}}$ are defined in (2.19).

Now, assume that EP $\{0\}$ of UNS (2.1) is $\mathrm{CWAS}_{\boldsymbol{\gamma}}$, but it is not CWEAS. As $\operatorname{EP}\{0\}$ of UNS (2.1) is not CWEAS (in accordance with our assumption), it results that there exists no $\boldsymbol{\alpha} \in \mathbb{R}^{n}, \boldsymbol{\alpha}>0$, such that $\overline{\mathbf{g}}(\boldsymbol{\alpha})<0$ and $\tilde{\mathbf{g}}(\boldsymbol{\alpha})<0$.

On the other hand, the EP $\{0\}$ of UNS (2.1) is $\mathrm{CWAS}_{\boldsymbol{\gamma}}$, meaning that (according to Theorem 4.3) there exists at least one $\boldsymbol{\gamma}(t)>0$ with $\lim _{t \rightarrow \infty} \boldsymbol{\gamma}(t)=0$, which is a PS of DI (2.18). It follows that there exists at least one point $t^{*} \in\left[t_{0}, \infty\right)$ for which we can write

$$
\begin{aligned}
& \overline{\mathcal{g}}_{i}\left(\boldsymbol{\gamma}\left(t^{*}\right)\right) \leq \dot{\gamma}_{i}\left(t^{*}\right)<0, \quad i=1, \ldots, n, \\
& \tilde{\mathcal{g}}_{i}\left(\boldsymbol{\gamma}\left(t^{*}\right)\right) \leq \dot{\gamma}_{i}\left(t^{*}\right)<0, \quad i=1, \ldots, n,
\end{aligned}
$$

and, moreover, (since $\boldsymbol{\gamma}(t)>0, t \in\left[t_{0}, \infty\right)$ ) that there exists at least one $\boldsymbol{\alpha}$, namely, $\boldsymbol{\alpha}=\boldsymbol{\gamma}\left(t^{*}\right)>0$, such that both inequalities $\overline{\mathbf{g}}(\boldsymbol{\alpha})<0$ and $\tilde{\mathbf{g}}(\boldsymbol{\alpha})<0$ are satisfied.

We have obviously reached a contradiction due to our assumption that $\operatorname{EP}\{0\}$ of UNS (2.1) is not CWEAS. This means that our assumption is false and $\operatorname{EP}\{0\}$ of UNS (2.1) is CWEAS.

The nonlinear algebraic inequalities (5.5) can be written compactly in a matrix form, using norm $\infty$, by considering the square matrices $\overline{\mathbf{M}}, \tilde{\mathbf{M}} \in \mathbb{R}^{n \times n}$ with the following entries:

$$
(\overline{\mathbf{M}})_{i j}=\frac{\bar{c}_{i j} \alpha_{j}^{p_{i j}}}{\alpha_{i}}, \quad(\tilde{\mathbf{M}})_{i j}=\frac{\tilde{c}_{i j} \alpha_{j}^{p_{i j}}}{\alpha_{i}}
$$

and a positive real number:

$$
s>\left|\hat{c}_{i i}\right|, \quad i=1, \ldots, n .
$$


THEOREM 5.7. EP\{0\} of UNS (2.1) is CWEAS if and only if there exist $\alpha_{i}>0$, $i=1, \ldots, n$, and $r<0$ such that

$$
\max \left\{\|\overline{\mathbf{M}}+s \mathbf{I}\|_{\infty},\|\tilde{\mathbf{M}}+s \mathbf{I}\|_{\infty}\right\} \leq r+s
$$

PROoF. Taking $s$ as in (5.15), inequalities (5.5) can be rewritten, equivalently (since $p_{i i}=1$ ), in the following form:

$$
\begin{aligned}
& \left(\bar{c}_{i i}+s\right)+\sum_{j=1}^{n} \frac{\bar{c}_{i j} \alpha_{j}^{p_{i j}}}{\alpha_{i}} \leq r+s, \quad i=1, \ldots, n, \\
& \left(\tilde{c}_{i i}+s\right)+\sum_{j=1}^{n} \frac{\widetilde{c}_{i j} \alpha_{j}^{p_{i j}}}{\alpha_{i}} \leq r+s, \quad i=1, \ldots, n,
\end{aligned}
$$

which, using a matrix formulation, means exactly (5.16) because all the entries of matrices $\overline{\mathbf{M}}+s \mathbf{I}$ and $\tilde{\mathbf{M}}+s \mathbf{I}$ are nonnegative.

THEOREM 5.8. EP\{0\} of UNS (2.1) is CWEAS if and only if there exist $\alpha_{i}>0$, $i=1, \ldots, n$, and $r<0$ such that

$$
\max \left\{\|\overline{\mathbf{M}}+s \mathbf{I} \mid\|_{\infty},\|\tilde{\mathbf{M}}+s \mathbf{I}\|_{\infty}\right\}<s .
$$

PROOF. It is a direct consequence of Theorem 5.7 due to the negativity of $r$.

REMARK 5.9. Theorems 5.7 and 5.8 present the advantage of a more tractable formulation from the computational point of view than inequalities (5.5) and (5.6), respectively. Thus, the determination of $\alpha_{i}, i=1, \ldots, n$, and $r$ can be approached as a nonlinear optimization problem with adequate constraints.

As already discussed in the general case of $\mathrm{CWAS}_{\gamma}$, it might be preferable to use a sufficient condition generated from DE (4.14) in Theorem 4.9. Therefore, consider the square matrix $\hat{\mathbf{P}} \in \mathbb{R}^{n \times n}$ with the following entries:

$$
(\hat{\mathbf{P}})_{i j}=\hat{c}_{i j} \varepsilon^{p_{i j}-1}, \quad \varepsilon>0,
$$

where $\hat{c}_{i j}, i, j=1, \ldots, n$, are defined by (4.15) in Theorem 4.9. Denote by $\lambda_{\max }(\hat{\mathbf{P}})$ the eigenvalue of $\hat{\mathbf{P}}$ (simple or multiple) with the greatest real part. As $\hat{c}_{i j} \geq 0$, $i \neq j, i, j=1, \ldots, n$, according to [9], $\lambda_{\max }(\hat{\mathbf{P}})$ is a real number.

THEOREM 5.10. If, for a given $\varepsilon>0$, matrix $\hat{\mathbf{P}}$ is Hurwitz stable, then the EP\{0\} of UNS (2.1) is CWEAS for some $0<\alpha_{i} \leq \varepsilon, i=1, \ldots, n$, and $\lambda_{\max }(\hat{\mathbf{P}}) \leq r<0$. 
Proof. Consider the square matrix $\hat{\mathbf{M}}$ whose entries are defined as

$$
(\hat{\mathbf{M}})_{i j}=\frac{\hat{c}_{i j} \alpha_{j}^{p_{i j}}}{\alpha_{i}}, \quad i=1, \ldots, n,
$$

where $\hat{c}_{i j}$ are defined according to (4.15). This means that we can write

$$
\max \left\{\|\overline{\mathbf{M}}+s \mathbf{I}\|_{\infty},\|\tilde{\mathbf{M}}+s \mathbf{I}\|_{\infty}\right\} \leq\|\hat{\mathbf{M}}+s \mathbf{I}\|_{\infty} .
$$

Now, for any $0<\alpha_{i} \leq \varepsilon$, we have

$$
\begin{aligned}
(\hat{\mathbf{M}})_{i j} & =\frac{\alpha_{j}\left(\hat{c}_{i j} \alpha_{j}^{p_{i j}-1}\right)}{\alpha_{i}} \leq \alpha_{j} \hat{c}_{i j} \varepsilon^{p_{i j}-1} / \alpha_{i} \\
& =\frac{\alpha_{i}(\hat{\mathbf{P}})_{i j}}{\alpha_{i}}=\alpha_{i}, \quad \forall i, j=1, \ldots, n, i \neq j, \\
(\hat{\mathbf{M}})_{i i} & =(\hat{\mathbf{P}})_{i i}, \quad i=1, \ldots, n,
\end{aligned}
$$

which can be globally written as

$$
\hat{\mathbf{M}} \leq \mathbf{D}^{-1} \hat{\mathbf{P}} \mathbf{D}
$$

where $\mathbf{D}=\operatorname{diag}\left\{\alpha_{1}, \ldots, \alpha_{n}\right\}$. It follows that

$$
\|\hat{\mathbf{M}}+s \mathbf{I}\|_{\infty} \leq\left\|\mathbf{D}^{-1} \hat{\mathbf{P}} \mathbf{D}+s \mathbf{I}\right\|_{\infty}
$$

where, for the right-hand side, we have

$$
\min _{\mathbf{D}>0}\left\|\mathbf{D}^{-1} \hat{\mathbf{P}} \mathbf{D}+s \mathbf{I}\right\|_{\infty}=\rho(\hat{\mathbf{P}}+s \mathbf{I})=\lambda_{\max }(\hat{\mathbf{P}})+s .
$$

It follows that, for any $0<\alpha_{i} \leq \varepsilon, i=1, \ldots, n$, we can write

$$
\max \left\{\|\overline{\mathbf{M}}+s \mathbf{I}\|_{\infty},\|\hat{\mathbf{M}}+s \mathbf{I}\|_{\infty}\right\} \leq \lambda_{\max }(\hat{\mathbf{P}})+s,
$$

which shows that, whenever $\hat{\mathbf{P}}$ is Hurwitz stable, condition (5.16) is met for $\lambda_{\max }(\hat{\mathbf{P}}) \leq r$. Thus, according to Theorem 5.7, EP\{0\} of UNS (2.1) is CWEAS for any $0<\alpha_{i} \leq \varepsilon$ and $\lambda_{\max }(\hat{\mathbf{P}}) \leq r$.

The advantage of Theorem 5.10 consists in a quick test on the stability of matrix $\hat{\mathbf{P}}$ which depends on a single parameter $\varepsilon>0$.

REMARK 5.11. According to Theorem 4.9, whenever inequalities (4.16) are satisfied, the existence of a positive $\varepsilon>0$, for which matrix $\hat{\mathbf{P}}$ is Hurwitz stable, represents a necessary and sufficient condition for the EP\{0\} of UNS (2.1) to be CWEAS. 
6. $\mathrm{CWAS}_{y}$ and CWEAS in linear approximation. The linear approximation of UNS (2.1) is an uncertain system with interval matrix, preserving only those elements $a_{i j} x_{j}^{p_{i j}}$ in $f_{i}(\mathbf{x}), i, j \geq 1, \ldots, n$, for which $p_{i j}=1$. Thus, it is necessary to have $p_{i j}=1, i=1, \ldots, n$, because, otherwise, the linear approximation of UNS (2.1) cannot be $\mathrm{CWAS}_{\boldsymbol{\gamma}}$ (or equivalently CWEAS), according to [9]. In other words, CWAS $y$ and CWEAS in linear approximation are equivalent concepts, and the linear approximation inherits the CWEAS property from UNS (2.1) as shown below.

THEOREM 6.1. Let $p_{i i}=1$ and $a_{i i}^{+}<0, i=1, \ldots, n$. EP\{0\} of UNS (2.1) is CWEAS if and only if the linear approximation of UNS (2.1) is CWEAS.

Proof. UNS (2.1) in the first approximation is described by the equations

$$
\dot{x}_{i}=\sum_{\substack{j=1 \\ p_{i j}=1}}^{n} a_{i j} x_{j}^{p_{i j}}, \quad i=1, \ldots, n,
$$

which, according to [9], is CWEAS if and only if the square matrix $\mathbf{B} \in \mathbb{R}^{n \times n}$, built with the common coefficients of $\bar{g}_{i}$ and $\tilde{g}_{i}$ in (2.19) as

$$
\text { (B) })_{i j}=b_{i j}= \begin{cases}\bar{c}_{i j}=\widetilde{c}_{i j}, & \text { if } p_{i j}=1, \\ 0, & \text { if } p_{i j} \geq 2,\end{cases}
$$

is Hurwitz stable. Equivalently, there exist $\alpha_{i}>0, i=1, \ldots, n$, such that the following inequalities are true:

$$
\sum_{j=1}^{n} \frac{b_{i j} \alpha_{j}}{\alpha_{i}}<0, \quad i=1, \ldots, n .
$$

NECESSITY. If EP $\{0\}$ of UNS (2.1) is CWEAS, then the above inequalities are fulfilled because

$$
\sum_{j=1}^{n} \frac{b_{i j} \alpha_{j}}{\alpha_{i}} \leq \max \left\{\sum_{j=1}^{n} \frac{\bar{c}_{i j} \alpha_{j}^{p_{i j}}}{\alpha_{i}}, \sum_{j=1}^{n} \frac{\hat{c}_{i j} \alpha_{j}^{p_{i j}}}{\alpha_{i}}\right\}, \quad i=1, \ldots, n .
$$

SUFFICIENCY. If UNS (2.1) in the first approximation is CWEAS, then there exists a small $q>0$ such that the inequalities

$$
\sum_{j=1}^{n} \frac{b_{i j} \alpha_{j}}{\alpha_{i}}<-q, \quad i=1, \ldots, n
$$

are satisfied for some $\alpha_{i}>0, i=1, \ldots, n$. We can choose (because of linearity) a set $\left\{\alpha_{1}^{*}, \ldots, \alpha_{n}^{*}\right\}$ with all $\alpha_{i}^{*} \geq 1, i=1, \ldots, n$. Denote by $\alpha_{\max }^{*}$ and $\alpha_{\min }^{*}$ the maximum and minimum value, respectively, for the set $\left\{\alpha_{1}^{*}, \ldots, \alpha_{n}^{*}\right\}$. Obviously, any other set of $\alpha_{i}, i=1, \ldots, n$, with $\alpha_{i}=\eta \alpha_{i}^{*}, \eta>0$, satisfies the above inequality. 
Denote by $c$ the maximum value of the coefficients $\bar{c}_{i j}$ and $\tilde{c}_{i j}$ for $p_{i j} \geq 2$ and by $p$ the maximum value of the $p_{i j} \geq 2$ for all $i, j=1, \ldots, n$. Thus, for any $\alpha_{i}=\eta \alpha_{i}^{*}, \eta \in(0,1]$, we can write

$$
\begin{aligned}
\sum_{\substack{j=1 \\
p_{i j} \geq 2}}^{n} \frac{\bar{c}_{i j} \alpha_{j}^{p_{i j}}}{\alpha_{i}} & =\sum_{\substack{j=1 \\
p_{i j} \geq 2}}^{n} \bar{c}_{i j}\left[\eta^{p_{i j}-1}\left(\alpha_{j}^{*}\right)^{p_{i j}-1}\right]\left(\frac{\alpha_{j}^{*}}{\alpha_{i}^{*}}\right) \\
& \leq \sum_{\substack{j=1 \\
p_{i j} \geq 2}}^{n} c\left[\eta^{p_{i j}-1}\left(\alpha_{j}^{*}\right)^{p_{i j}-1}\right] \frac{\alpha_{\max }^{*}}{\alpha_{\min }^{*}} \\
& \leq c \frac{\alpha_{\max }^{*}}{\alpha_{\min }^{*}} \sum_{j=1}^{n} \eta\left(\alpha_{\max }^{*}\right)^{p-1} \\
& =\eta c n \frac{\left(\alpha_{\max }^{*}\right)^{p}}{\alpha_{\min }^{*}}, \quad i=1, \ldots, n,
\end{aligned}
$$

and, similarly,

$$
\sum_{\substack{j=1 \\ p_{i j} \geq 2}}^{n} \frac{\tilde{c}_{i j} \alpha_{j}^{p_{i j}}}{\alpha_{i}} \leq \eta c n \frac{\left(\alpha_{\max }^{*}\right)^{p}}{\alpha_{\min }^{*}}, \quad i=1, \ldots, n .
$$

Now, taking $\eta^{*}=q \alpha_{\min }^{*} / c n\left(\alpha_{\max }^{*}\right)^{p}<1$ for $\alpha_{i}=\eta^{*} \alpha_{i}^{*}, i=1, \ldots, n$, we can write

$$
\sum_{\substack{j=1 \\ p_{i j} \geq 2}}^{n} \frac{\bar{c}_{i j} \alpha_{j}^{p_{i j}}}{\alpha_{i}} \leq q, \quad \sum_{\substack{j=1 \\ p_{i j} \geq 2}}^{n} \frac{\tilde{c}_{i j} \alpha_{j}^{p_{i j}}}{\alpha_{i}} \leq q,
$$

and, moreover,

$$
\sum_{j=1}^{n} \frac{\bar{c}_{i j} \alpha_{j}^{p_{i j}}}{\alpha_{i}}<0, \quad \sum_{j=1}^{n} \frac{\tilde{c}_{i j} \alpha_{j}^{p_{i j}}}{\alpha_{i}}<0,
$$

meaning the fulfillment of the necessary and sufficient condition for $\operatorname{EP}\{0\}$ of UNS (2.1) to be CWEAS that was formulated in Theorem 5.5.

REMARK 6.2. The equivalence stated by the previous theorem should be understood just in qualitative terms around the $\operatorname{EP}\{0\}$ since, for the linear approximation of UNS (2.1), CWEAS is a global property (due to the linearitysee [9]), whereas both direct and converse proofs in Theorem 6.1 refer to a local property of UNS (2.1).

7. Examples. The following two examples illustrate the applicability of FI theory to a componentwise investigation of the dynamics around the EPs of 
UNSs, able to reveal characteristics which remain hidden for the standard tools of stability analysis.

EXAMPLE 7.1. Consider the second-order UNS described by the equations

$$
\dot{x}_{1}=[-5,-2] x_{1}+[4,7] x_{2} ; \quad \dot{x}_{2}=[-3,-1] x_{1}^{3} .
$$

$\mathrm{EP}\{0\}$ of UNS (2.1) is globally asymptotically stable. Indeed, for any constant value belonging to the interval-type coefficients, we get a system of the following form:

$$
\dot{x}_{1}=a_{11} x_{1}+a_{12} x_{2} ; \quad \dot{x}_{2}=a_{21} x_{1}^{3},
$$

where $a_{11} \in[-5,-2], a_{12} \in[4,7]$, and $a_{21} \in[-3,-1]$. If we take the Lyapunov function

$$
V(x)=-\frac{1}{4 a_{11}} x_{1}^{4}+\frac{a_{12}}{2 a_{11} a_{21}} x_{2}^{2}>0 \quad \forall x_{1}, x_{2} \neq 0,
$$

it results $\dot{V}(x)=-x_{1}^{4}<0$ for any nontrivial state-space trajectory, that is, the $\mathrm{EP}\{0\}$ is globally asymptotically stable.

However, the $\operatorname{EP}\{0\}$ of this UNS (regarded as a family of systems) cannot be $\mathrm{CWAS}_{\boldsymbol{y}}$ because the coefficient of $x_{2}$ in the second equation is 0 , violating the necessary condition formulated in Theorem 4.5. Moreover, none of the nonlinear constant systems belonging to the family generated by this UNS is CWAS (since Theorem 4.5 is violated for all possible constant values belonging to the interval-type coefficients).

A graphical representation can be given for any set of constant values of the coefficients corresponding to the UNS considered in this example, showing that the state trajectories leave any stationary TDRS. For instance, Figure 7.1 plots the evolution of the vertices of a TDRS with $\gamma_{1}(t)=1, \gamma_{2}(t)=2$ in the right-hand side of equality (2.3) (solid line), and the evolution of the four state trajectories initialized in the vertices of this TDRS at $t=0$, for $a_{11}=-2, a_{12}=$ 7 , and $a_{21}=-1$ (dotted line).

The first example clearly shows that the $\mathrm{CWAS}_{\boldsymbol{\gamma}}$ concept is far from triviality once the UNS is globally AS, but the $\mathrm{CWAS}_{\boldsymbol{\gamma}}$ condition cannot be satisfied by any member of the family generated by this UNS. Unlike this example, the second one demonstrates the existence of the CWEAS property for a UNS whose $\operatorname{EP}\{0\}$ is only locally AS.

EXAMPLE 7.2. Consider the second-order UNS described by the equations

$$
\dot{x}_{1}=[-4,-2] x_{1}+[-1,2] x_{2}^{2} ; \quad \dot{x}_{2}=[-3,1] x_{1}^{2}+[-6,-5] x_{2} .
$$




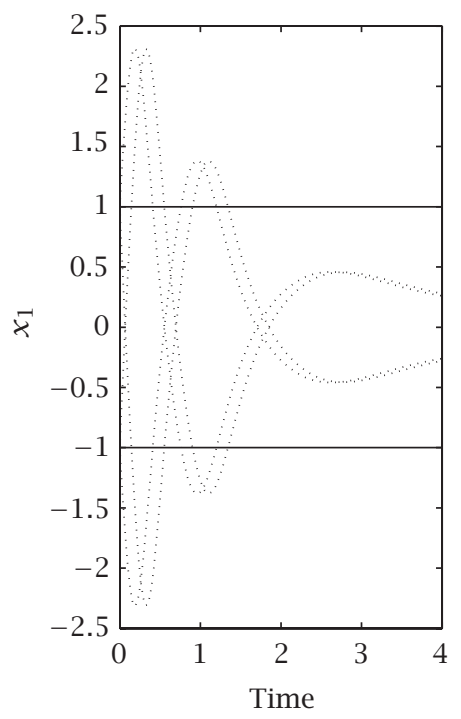

(a)

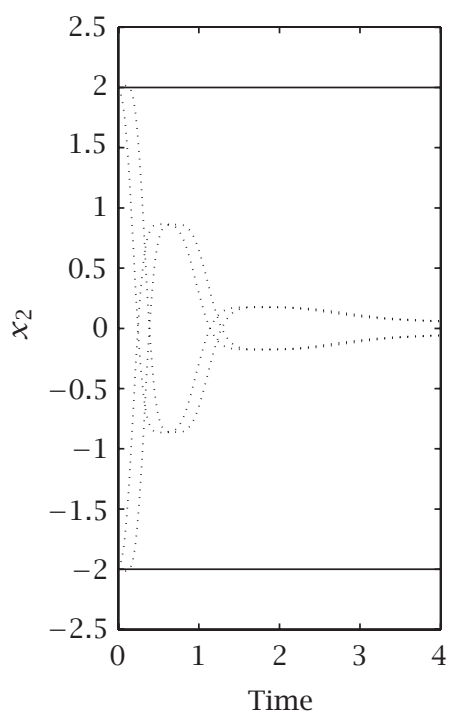

(b)

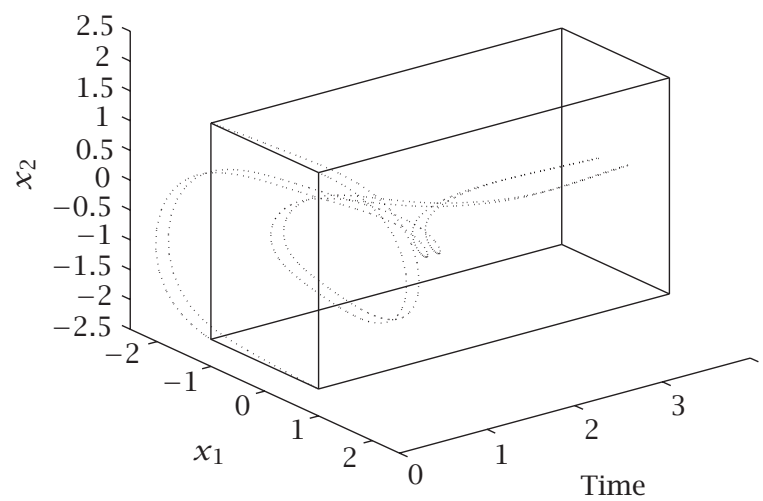

(c)

FIGURE 7.1. Plots corresponding to the UNS considered in Example 7.1 for constant coefficients $a_{11}=-2, a_{12}=7$, and $a_{21}=-1$ and TDRS with $\gamma_{1}(t)=1, \gamma_{2}(t)=2$. Solid line is used for the evolution of the vertices of TDRS and dotted line for the evolution of the four selected state trajectories. (a) A 2D visualisation for state variable $x_{1}$ versus time. (b) A 2D visualisation for state variable $x_{2}$ versus time. (c) A 3D visualisation. 
We immediately see that $p_{11}=p_{22}=1$ and $a_{11}^{+}=-2<0$, and $a_{22}^{+}=-5<0$, and, therefore, the $\mathrm{CWAS}_{\boldsymbol{\gamma}}$ condition for $\mathrm{EP}\{0\}$ of UNS is equivalent to CWEAS (according to Theorem 5.6). Thus, we investigate the compatibility of the following nonlinear algebraic inequalities:

$-2 \alpha_{1}+2 \alpha_{2}^{2}<0, \quad \alpha_{1}^{2}-5 \alpha_{2}<0, \quad$ corresponding to (5.10) in Theorem 5.5,

$-2 \alpha_{1}+\alpha_{2}^{2}<0, \quad 3 \alpha_{1}^{2}-5 \alpha_{2}<0, \quad$ corresponding to (5.11) in Theorem 5.5,

which can be reduced to

$$
-2 \alpha_{1}+2 \alpha_{2}^{2}<0, \quad 3 \alpha_{1}^{2}-5 \alpha_{2}<0,
$$

or, equivalently, to

$$
\alpha_{2}^{2}<\alpha_{1} ; \quad \frac{3}{5} \alpha_{1}^{2}<\alpha_{2}
$$

It is obvious that there exist $\alpha_{1}>0, \alpha_{2}>0$ ensuring compatibility. Hence, $\mathrm{EP}\{0\}$ of this UNS is CWEAS.

The CWEAS problem of EP\{0\} of this UNS can also be approached using Theorem 6.1 and the linear approximation of UNS

$$
\dot{x}_{1}=[-4,-2] x_{1} ; \quad \dot{x}_{2}=[-6,-5] x_{2},
$$

which yields the linear inequalities

$$
-2 \alpha_{1}<0 ; \quad-5 \alpha_{2}<0 .
$$

The solution set $\left\{\alpha_{1}, \alpha_{2}\right\}$ previously obtained for the UNS is, obviously, smaller than the solution set got for the linear approximation. However, each solution $\left\{\alpha_{1}, \alpha_{2}\right\}$ of the linear approximation, multiplied by an adequately small positive constant (i.e., $\eta$ in the proof of Theorem 6.1) becomes a solution of the initial UNS.

If a set of constant values is selected for the coefficients of the UNS in this example, we can construct relevant graphical plots for the dynamics under flow-invariance constraints. For instance, if $a_{11}=-2, a_{22}=2, a_{21}=1$, and $a_{22}=-5$, then a TDRS ensuring CWEAS for the nonlinear system is obtained by taking $\alpha_{1}=1, \alpha_{2}=0.74$, and $r=-0.9$ in the right-hand side of equality (5.1). Figure 7.2 plots the evolution of the vertices of TDRS (solid line) and of the four state trajectories initialized in the vertices of TDRS at $t=0$ (dotted line).

8. Conclusions. Given UNS (2.1), we have defined the FI of TDRS $\mathbf{H}(t)$ (2.3) with respect to this UNS (Definition 2.1), and we have derived a characterization of FI in terms of differential inequalities (Theorem 2.2). Two results (Theorems 2.3 and 2.4) emphasize the equivalence between the existence of TDRSs 


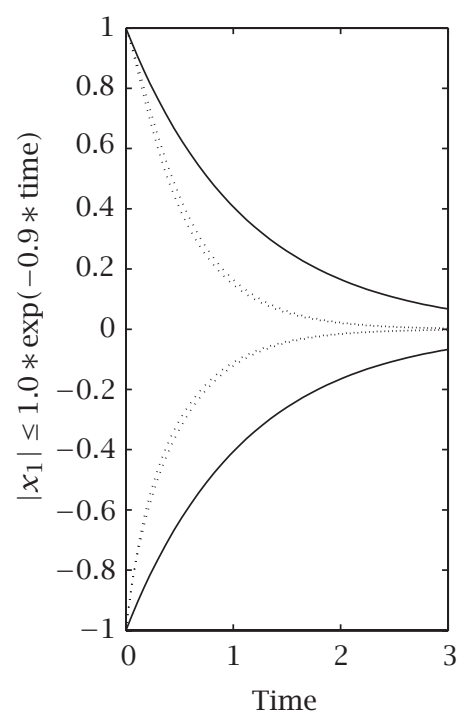

(a)

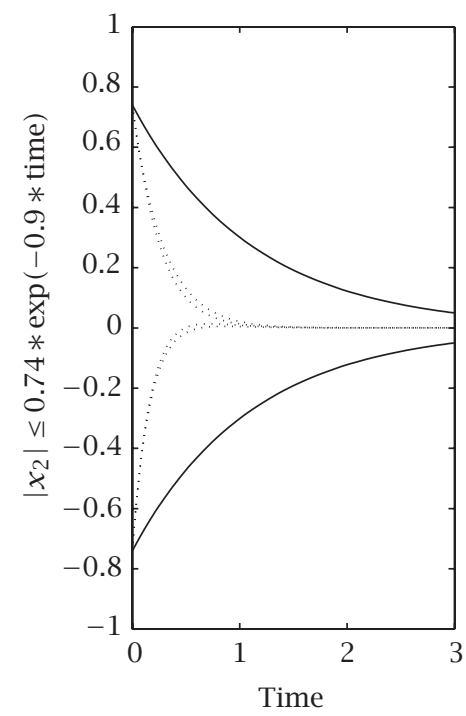

(b)

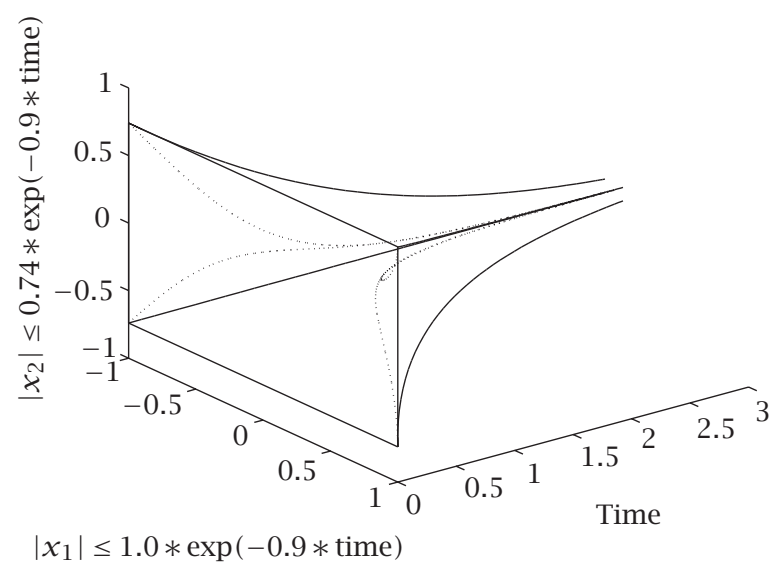

(c)

FIGURE 7.2. Plots corresponding to the UNS considered in Example 7.2 for constant coefficients $a_{11}=-2, a_{22}=2, a_{21}=1$, and $a_{22}=$ -5 . Solid line is used for the evolution of the vertices of TDRS with $\gamma_{1}(t)=e^{-0.9 t}, \gamma_{2}(t)=0.74 e^{-0.9 t}$ and dotted line for the evolution of the four selected state trajectories. (a) A 2D visualisation for state variable $x_{1}$ versus time. (b) A 2D visualisation for state variable $x_{2}$ versus time. (c) A 3D visualisation. 
possessing the FI property and the existence of PSs for differential inequalities (2.7) and (2.19), respectively. This equivalence allows, further, exploring the structure of the whole family of TDRSs which are FI with respect to UNS (2.1) (Theorems 3.4, 3.5, and 3.7).

The FI concept provides basic tools for dealing with $\mathrm{CWAS}_{y}$ (Definition 4.1) as a special type of AS, where the evolution of the state variables approaching the $\mathrm{EP}\{0\}$ is characterized individually (unlike the standard AS, which relies on a global knowledge stated in terms of norms). Thus, after some intermediary results (Theorems 4.3, 4.4, and 4.5), we prove (Theorem 4.7) that the CWAS of $\mathrm{EP}\{0\}$ for UNS (2.1) is equivalent to the standard AS of EP $\{0\}$ for DE (3.1). Moreover, we show that a sufficient (and, in some cases, also necessary) condition is the standard AS of $\mathrm{EP}\{0\}$ for DE (4.14), whose form is simpler than DE (3.1) (Theorem 4.9).

Supplementary requirements for the individual evolution of the state variables approaching the EP\{0\} allow introducing CWEAS (Definition 5.1), representing a particular type of $\mathrm{CWAS}_{\gamma}$, which may exist only for specific structures of UNS (2.1) (Theorem 5.3). These requirements make it possible to formulate algebraic conditions that are necessary and sufficient for the CWEA $S_{\gamma}$ of $\operatorname{EP}\{0\}$ of UNS (2.1) (Theorems 5.4, 5.5, 5.6, and 5.7). It is shown that, whenever the structure of UNS (2.1) permits the existence of CWEAS, $\mathrm{CWAS}_{\gamma}$ is equivalent to CWEAS. Simplified algebraic conditions can be derived (Theorem 5.10) as sufficient (and, in some cases, also necessary) conditions. Finally, for all those structures of UNS (2.1) allowing the existence of CWEAS, we reveal the link between the CWEAS of EP\{0\} for UNS (2.1) and the CWEAS of the linear system with interval matrix, representing the first approximation of UNS (2.1). Two examples illustrate the applicability of FI theory to the componentwise investigation of the dynamics around the EPs of UNSs, able to reveal characteristics, which remain hidden for the standard tools of stability analysis.

\section{REFERENCES}

[1] H. Brezis, On a characterization of flow-invariant sets, Comm. Pure Appl. Math. 23 (1970), 261-263.

[2] M. G. Crandall, A generalization of Peano's existence theorem and flow invariance, Proc. Amer. Math. Soc. 36 (1972), 151-155.

[3] A. Hmamed, Componentwise stability of continuous-time delay linear systems, Automatica J. IFAC 32 (1996), no. 4, 651-653.

[4] M. Hukuhara, Sur la théorie des équations différentielles ordinaires, J. Fac. Sci. Univ. Tokyo Sect. I 7 (1958), 483-510 (French).

[5] R. H. Martin Jr., Differential equations on closed subsets of a Banach space, Trans. Amer. Math. Soc. 179 (1973), 399-414.

[6] G. Morosanu, Differential Equations. Applications, Editura Academiei, Bucuresti, 1989 (Romanian).

[7] D. Motreanu and N. H. Pavel, Tangency, Flow Invariance for Differential Equations, and Optimization Problems, Monographs and Textbooks in Pure and Applied Mathematics, vol. 219, Marcel Dekker, New York, 1999. 
[8] M. Nagumo, Über die Lage der Integralkurven gewöhnlicher Differentialgleichungen, Proc. Phys.-Math. Soc. Japan (3) 24 (1942), 551-559 (German).

[9] O. Pastravanu and M. Voicu, Flow-invariant rectangular sets and componentwise asymptotic stability of interval matrix systems, Proc. of the 5th European Control Conference (ECC '99) (Karlsruhe), 1999, CDROM.

[10] _ Robustness analysis of componentwise asymptotic stability, Proc. of the 15-th World Congress of IMACS (Lausanne), 2000, CDROM.

[11] N. H. Pavel, Differential Equations, Flow Invariance and Applications, Research Notes in Mathematics, vol. 113, Pitman, Massachusetts, 1984.

[12] M. Voicu, Componentwise asymptotic stability of linear constant dynamical systems, IEEE Trans. Automat. Control 29 (1984), no. 10, 937-939.

[13] _ Free response characterization via flow invariance, preprints of the 9-th World Congress of IFAC (Budapest), vol. 5, 1984, pp. 12-17.

[14] On the application of the flow invariance method in control theory and design, preprints of the 10-th World Congress of IFAC (Munich), vol. 8, 1987, pp. 364-369.

Octavian Pastravanu: Department of Automatic Control and Industrial Informatics, "Gh. Asachi" Technical University of Iasi, Boulevard D Mangeron 53A, 6600 Iasi, Romania

E-mail address: opastrav@ac.tuiasi .ro

Mihail Voicu: Department of Automatic Control and Industrial Informatics, "Gh. Asachi” Technical University of Iasi, Boulevard D Mangeron 53A, 6600 Iasi, Romania

E-mail address: mvoicu@ac . tuiasi . ro 


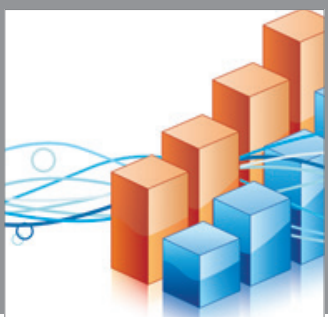

Advances in

Operations Research

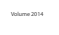

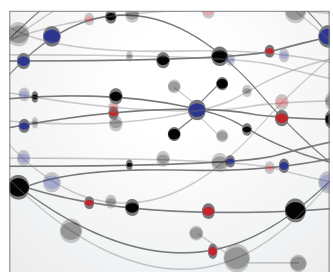

\section{The Scientific} World Journal
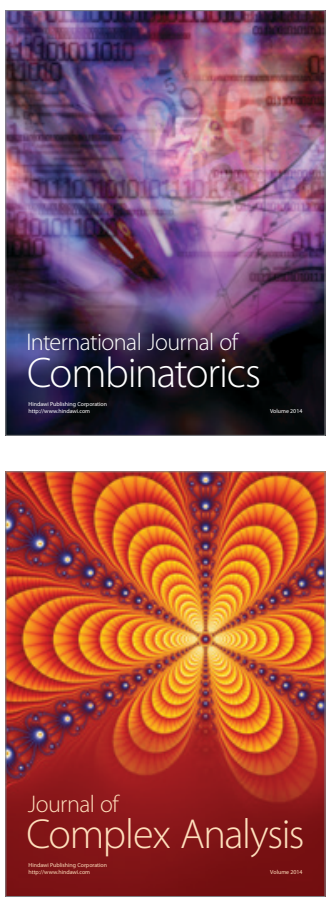

International Journal of

Mathematics and

Mathematical

Sciences
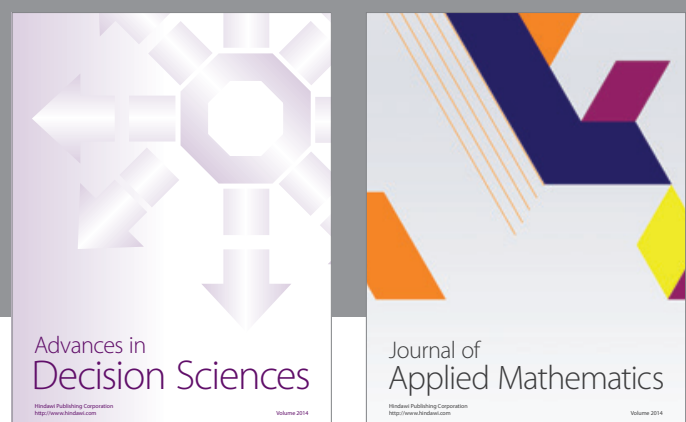

Journal of

Applied Mathematics
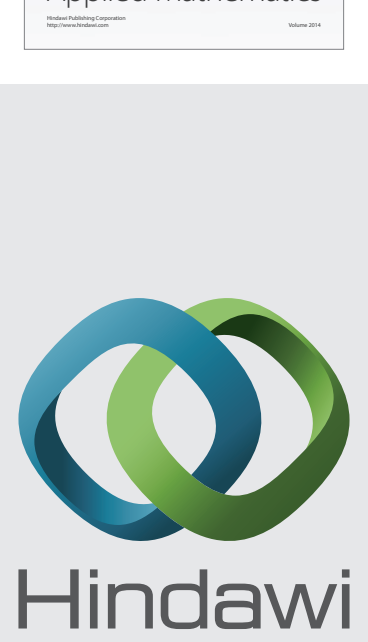

Submit your manuscripts at http://www.hindawi.com
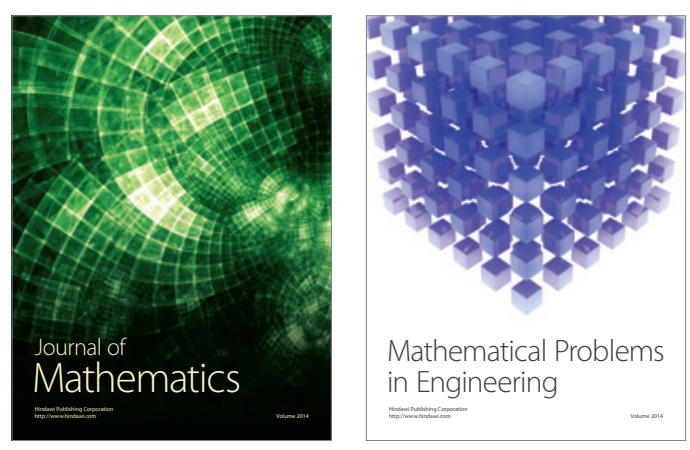

Mathematical Problems in Engineering
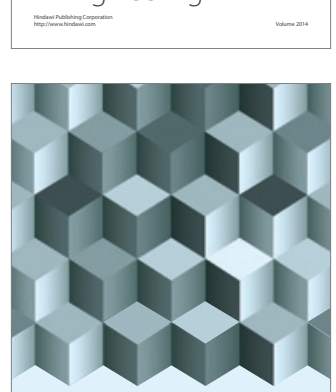

Journal of

Function Spaces
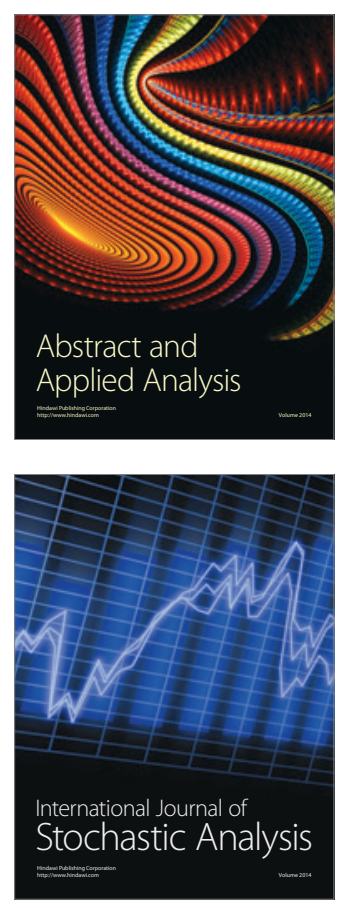

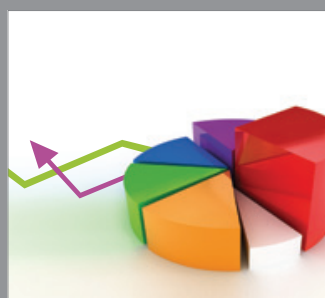

ournal of

Probability and Statistics

Promensencen
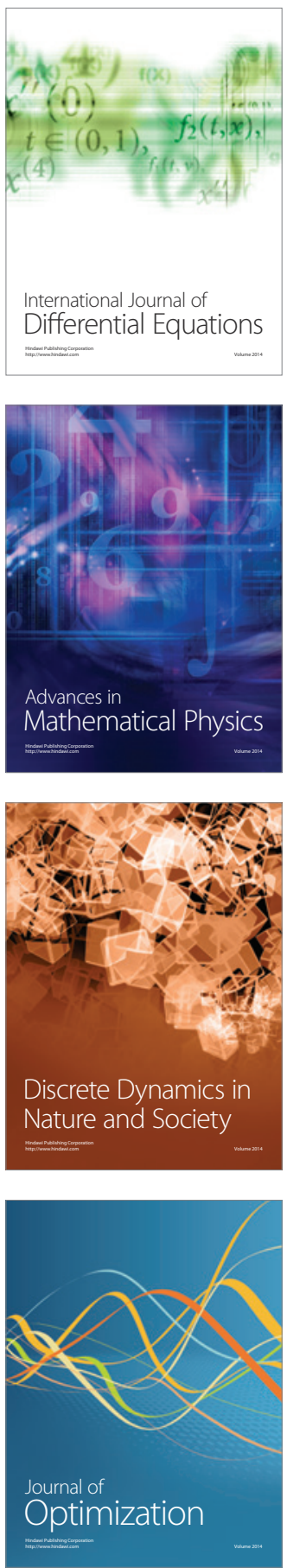